KA-TP-08-2001

hep-ph/0103009

\title{
Regularization and supersymmetry-restoring counterterms in supersymmetric QCD
}

\author{
W. Hollik and D. Stöckinger \\ Institut für Theoretische Physik, Universität Karlsruhe, D-76128 Karlsruhe, Germany \\ e-mail: hollik@particle.physik.uni-karlsruhe.de, ds@particle.physik.uni-karlsruhe.de
}

October 25, 2018

\begin{abstract}
We calculate symmetry-restoring counterterms in supersymmetric QCD at the one-loop level. First we determine loop corrections to the supersymmetry and gauge transformations and find counterterms in such a way that the symmetry algebra holds at the one-loop level. Then these results are used to derive the symmetry-restoring counterterms to all trilinear interactions. In order to obtain unique results it is crucial to use the Slavnov-Taylor identity, which does not only contain supersymmetric and gauge Ward identities but also describes the symmetry algebra. In dimensional regularization this procedure yields unique non-zero values for the counterterms. In contrast, in dimensional reduction we find that no non-symmetric counterterms are needed, neither for the symmetry transformations nor for the physical interactions. For the considered cases this result constitutes a definite test of the supersymmetry and gauge invariance of the scheme.
\end{abstract}

\section{Introduction}

It has been a longstanding problem that dimensional regularization (DReg) breaks supersymmetry. In general, this breaking necessitates the calculation of compensating, supersymmetry-restoring counterterms. An efficient solution would be provided by a manifestly supersymmetric and gauge invariant regularization, but no such regularization is known. A practically useful scheme is dimensional reduction (DRed), which is however mathematically inconsistent and thus cannot work at all orders [1,2]. Still it has been shown that several supersymmetric Ward identities are satisfied in DRed [3] and that, as long as the inconsistencies of DRed do not play a role, DRed is related to DReg by a coupling constant redefinition and thus leads to equivalent results [4].

At the same time there were severe difficulties to find the correct way to renormalize supersymmetric gauge theories in a regularization-independent way. In the WessZumino gauge, which is the gauge almost exclusively used in practical calculations, the usual way of treating global symmetries by Ward identities was shown to fail for supersymmetry (for an account see [5]).

By now these difficulties have been solved for exact supersymmetry [6, 7, 8] as well as for the case of softly broken supersymmetry [9, 10]. In particular, a consistent set of symmetry identities (Ward- and Slavnov-Taylor identi- ties) has been found that provides an unambiguous definition of the theories.

There is a major difference between the supersymmetric Slavnov-Taylor identity found in [6] and the Ward identities considered in [3,5]. For Green functions, the Ward identities can be written as

$$
\delta_{\text {susy }}\left\langle T \phi_{1} \ldots \phi_{n}\right\rangle=\left\langle T \Delta \phi_{1} \ldots \phi_{n}\right\rangle,
$$

where $\delta_{\text {susy }}$ denotes the infinitesimal supersymmetry transformations of the fields and the composite operator $\Delta$ is due to the supersymmetry breaking of the gauge fixing in the Wess-Zumino gauge. The symmetry transformations are generally non-linear composite operators receiving quantum corrections that have to be renormalized, and the meaning of (II) is the invariance of the theory under these renormalized transformations. But the invariance expressed by (11) does not necessarily correspond to supersymmetry. It only does if the renormalized transformations satisfy the supersymmetry algebra, and only the Slavnov-Taylor identity contains both the invariance of the theory and the algebra. Indeed, the SlavnovTaylor identity determines the structure constants of the symmetry algebra and thus governs the renormalization of the symmetry operators. As a consequence, only the Slavnov-Taylor identity yields unique results for the nonsupersymmetric counterterms or provides definite tests of the supersymmetry of a calculation. 
The purpose of this article is twofold. First we develop the Feynman rules involving all the ghost fields and external fields that appear in the Slavnov-Taylor identity. Then we use the Slavnov-Taylor identity to determine supersymmetry-restoring counterterms in dimensional regularization and to check whether a given regularization such as DRed preserves supersymmetry at oneloop order. We do this in supersymmetric QCD with soft breaking, a model of particular phenomenological interest with generally large quantum corrections. The SlavnovTaylor identity not only describes supersymmetry but also gauge invariance. Taking into account all its consequences for the symmetries and the symmetry algebra, we calculate all symmetry-restoring counterterms to the trilinear interactions and the required counterterms to the symmetry transformations.

Section 2 sets the basis for our calculations. Since the Slavnov-Taylor identity is the key relation, it is briefly reviewed, with emphasis on the meaning of the involved ghost fields and of the special cases we need in later course.

In sec. 3 we derive and discuss the counterterms to all trilinear interactions and the necessary counterterms to the symmetry transformations; in sec. A we present our conclusions. In App. B the Feynman rules are listed, in particular the ones involving the ghost fields. We need them to calculate the loop corrections to the symmetry transformation operators. The rest of the appendices is devoted to the explicit form of the Lagrangian, the BRS transformations, and the one-loop results of the necessary vertex functions.

\section{The Slavnov-Taylor identity of supersymmetric QCD}

In this section we briefly review the Slavnov-Taylor identity of supersymmetric QCD [6, 0, 8, 9, 10, since it is the mathematical expression for the symmetries of the model. Its knowledge is necessary for testing whether a regularization preserves the symmetries as well as for the determination of supersymmetry-restoring counterterms.

\subsection{BRS invariance and gauge fixing}

The usual gauge fixing term of supersymmetric QCD

$$
\mathcal{L}_{\text {fix }}=-\frac{1}{2 \xi}\left(\partial_{\mu} G_{a}^{\mu}\right)^{2}
$$

does not only break gauge invariance but also supersymmetry because it contains only the gluon $G_{a}^{\mu}$ but not the gluino. As a consequence it is very useful to treat gauge invariance and supersymmetry simultaneously using combined BRS transformations. Then not only the FaddeevPopov ghosts are needed but also supersymmetry ghosts.

Actually, three kinds of ghost fields are introduced: the Faddeev-Popov ghosts $c_{a}(x)$ that correspond to gauge transformations and are fermionic scalar fields, supersymmetry ghosts $\epsilon$ (bosonic Majorana spinors), and translational ghosts $\omega^{\mu}$ (fermionic vectors). Translations cannot be treated separately since they arise as anticommutators of supersymmetry transformations. Among these ghost fields only the $c_{a}(x)$ are dynamical fields, whereas $\epsilon$ and $\omega^{\mu}$ are space-time independent constants since the corresponding symmetry transformations are global.

The explicit form of the BRS operator $s$ is listed in the appendix. As a crucial property of the BRS transformations, the transformations of the ghosts are determined by the structure constants of the symmetry algebra. Hence, knowing these BRS transformations is equivalent to knowing all the (anti-)commutators of the symmetry generators. It is this property that renders the BRS operator nilpotent, so that $s^{2}=0$ up to equations of motions.

Using the nilpotency of the BRS operator it is possible to write down a BRS-invariant gauge-fixing and ghost term: For the usual $\xi$-gauge one has

$$
\begin{aligned}
\Gamma_{\mathrm{fix}, \mathrm{gh}}= & \int d^{4} x \mathcal{L}_{\mathrm{fix}}, \mathrm{gh} \\
\mathcal{L}_{\mathrm{fix}, \mathrm{gh}}= & s\left[\bar{c}_{a}\left(f_{a}+\frac{\xi}{2} B_{a}\right)\right] \\
= & B_{a} f_{a}+\frac{\xi}{2} B_{a}^{2}-\bar{c}_{a} \partial_{\mu}\left(D^{\mu} c\right)_{a} \\
& -\bar{c}_{a} \partial^{\mu}\left(\bar{\epsilon} \gamma_{\mu} \tilde{g}_{a}\right)+\frac{1}{2} \xi i \bar{\epsilon} \gamma^{\nu} \epsilon\left(\partial_{\nu} \bar{c}_{a}\right) \bar{c}_{a},
\end{aligned}
$$

with $f_{a}=\partial_{\mu} G_{a}^{\mu}$ and the Faddeev-Popov antighosts $\bar{c}_{a}(x)$. For diagrammatic calculations it is customary to eliminate the auxiliary fields $B_{a}$, yielding the usual gauge fixing term (2). Note that the supersymmetry breaking of this gauge fixing term necessitates compensating terms involving the $\epsilon$ ghosts.

\subsection{Slavnov-Taylor identity}

At the tree level, BRS invariance can be expressed in the following way:

$$
0=\int d^{4} x s \varphi_{i} \frac{\delta \Gamma_{\mathrm{inv}}}{\delta \varphi_{i}},
$$

where $\Gamma_{\text {inv }}$ is a BRS invariant action and the sum runs over all fields of the model. At the quantum level, the BRS transformations have to be treated as composite operators. The non-linear composite operators receive loop corrections and have to be renormalized in the same way as the Green functions. It is most convenient to perform the renormalization of these composite symmetry operators together with the renormalization of the Lagrangian. To do that we couple the non-linear BRS transformations $s \varphi_{i}$ to external sources $Y_{i}$ according to the scheme

$$
\begin{aligned}
s \varphi_{i} & \longrightarrow \pm \frac{\delta \Gamma_{\mathrm{cl}}}{\delta Y_{i}}+\mathcal{O}\left(Y_{i}\right) \\
\left\langle s \varphi_{i}\right\rangle_{J} & \longrightarrow \pm \frac{\delta \Gamma}{\delta Y_{i}}+\mathcal{O}\left(Y_{i}\right)
\end{aligned}
$$

${ }^{1}$ The minus sign applies for complex conjugate fields. The reason is the reality of the BRS operator leading to the rule $(s B o s)^{\dagger}=s(B o s)^{\dagger},(s F e r)^{\dagger}=-s(F e r)^{\dagger}$. 
at the classical and at the quantum level. The resulting classical action $\Gamma_{\mathrm{cl}}$ is written down in App. A.2. $\Gamma$ denotes the renormalized effective action, the generating functional of one-particle irreducible vertex functions. 2 Clearly, the expectation values of products of operators differ from the products of the individual expectation values. This reflects the appearance of non-trivial loop corrections to the non-linear composite operators.

Now it is possible to write down the Slavnov-Taylor identity. It expresses the invariance under the loopcorrected BRS transformations (which incorporate gauge and supersymmetry transformations and translations) and the fact that the loop-corrected transformations still satisfy the desired symmetry algebra. The Slavnov-Taylor identity reads:

$$
\begin{aligned}
& S(\Gamma)=0 \\
& S(\Gamma)=S_{0}(\Gamma)+S_{\text {soft }}(\Gamma)
\end{aligned}
$$

with the part corresponding to unbroken supersymmetry,

$$
\begin{aligned}
S_{0}(\Gamma)= & \int\left(\frac{\delta \Gamma}{\delta Y_{G_{a \mu}}} \frac{\delta \Gamma}{\delta G_{a}^{\mu}}+\frac{\delta \Gamma}{\delta \tilde{g}_{a}} \frac{\delta \Gamma}{\delta \tilde{y}_{\tilde{g}} a}\right. \\
& +\sum_{k=L, R}\left(\frac{\delta \Gamma}{\delta \tilde{y}_{k}} \frac{\delta \Gamma}{\delta \tilde{q}_{k}}-\frac{\delta \Gamma}{\delta \tilde{y}_{k}^{\dagger}} \frac{\delta \Gamma}{\delta \tilde{q}_{k}^{\dagger}}\right) \\
& +\frac{\delta \Gamma}{\delta q} \frac{\delta \Gamma}{\delta \bar{y}}-\frac{\delta \Gamma}{\delta y} \frac{\delta \Gamma}{\delta \bar{q}} \\
& \left.+\frac{\delta \Gamma}{\delta Y_{c a}} \frac{\delta \Gamma}{\delta c_{a}}+s \bar{c}_{a} \frac{\delta \Gamma}{\delta \bar{c}_{a}}+s B_{a} \frac{\delta \Gamma}{\delta B_{a}}\right) \\
& +s \omega^{\mu} \frac{\delta \Gamma}{\delta \omega^{\mu}}
\end{aligned}
$$

and the part describing the soft breaking,

$$
\begin{aligned}
S_{\mathrm{soft}}= & \int\left(s a \frac{\delta \Gamma}{\delta a}+s a^{\dagger} \frac{\delta \Gamma}{\delta a^{\dagger}}+s \chi \frac{\delta \Gamma}{\delta \chi}\right. \\
& \left.+s f \frac{\delta \Gamma}{\delta f}+s f^{\dagger} \frac{\delta \Gamma}{\delta f^{\dagger}}\right) .
\end{aligned}
$$

In the Slavnov-Taylor operator all fields of the model appear: The gluons $G_{a}^{\mu}$ and the gluinos $\tilde{g}_{a}$, the quark $q$ and the squarks $\tilde{q}_{L, R}$ as well as the ghost fields, and the corresponding sources. Due to the squark mixing, the mass eigenstates $\tilde{q}_{1,2}$ are in general different from the interaction eigenstates $\tilde{q}_{L, R}$. We write the relation as follows,

$$
\begin{aligned}
& \tilde{q}_{k}=S_{k L} \tilde{q}_{L}+S_{k R} \tilde{q}_{R}, \\
& \tilde{y}_{k}=S_{k L}^{*} \tilde{y}_{L}+S_{k R}^{*} \tilde{y}_{R}
\end{aligned}
$$

with a unitary matrix $S$ diagonalizing the tree level squark mass matrix. $S_{\text {soft }}(\Gamma)$ involves the auxiliary chiral supermultiplet $\left(a, P_{L} \chi, \hat{f}=f+f_{0}\right)$ and its hermitian conjugate. The main property of this auxiliary multiplet is the constant piece $f_{0}$ that acts like a vacuum expectation value of $\hat{f}$ and generates the soft-breaking terms while permitting a fully supersymmetric formulation of the model.

\footnotetext{
${ }^{2}$ The sources $J_{i}=-\frac{\delta}{\delta \varphi_{i}}$ for the fields are to be understood as the usual sources in the functional integral.
}

\subsection{Important special cases}

For our later applications several special cases of the Slavnov-Taylor identity are particularly important. First it can be used to describe gauge invariance by taking the derivative with respect to the Faddeev-Popov ghost $c_{a}$ and setting all ghost fields and sources to zero ("gh=0"):

$$
\begin{aligned}
0 & =\frac{\delta S(\Gamma)}{\delta c_{a}} \\
\Rightarrow \quad 0 & =\frac{\delta \Gamma}{\delta c_{a} \delta Y_{i}} \frac{\delta \Gamma}{\delta \varphi_{i}}+\left.s \bar{c}_{b} \frac{\delta \Gamma}{\delta c_{a} \delta \bar{c}_{b}}\right|_{\mathrm{gh}=0} .
\end{aligned}
$$

The functions $\delta \Gamma / \delta c_{a} \delta Y_{i}$ are the loop-corrected gauge transformations of $\varphi_{i}$, and the last term in this identity is due to gauge fixing. Similarly, the Slavnov-Taylor identity can be used to describe supersymmetry by taking the derivative with respect to the supersymmetry ghost and setting all ghost fields and sources to zero:

$$
\begin{aligned}
0 & =\frac{\delta S(\Gamma)}{\delta \epsilon} \\
\Rightarrow \quad 0 & =\frac{\delta \Gamma}{\delta \epsilon \delta Y_{i}} \frac{\delta \Gamma}{\delta \varphi_{i}}+\left.\frac{\delta s \chi}{\delta \epsilon} \frac{\delta \Gamma}{\delta \chi}\right|_{\mathrm{gh}=0} .
\end{aligned}
$$

Here the first term is the supersymmetry transformation of $\varphi_{i}$, the last term is due to the soft supersymmetry breaking. When the auxiliary fields $a, \chi, f$ are set to zero, one has $\delta s \chi / \delta \epsilon=\sqrt{2}\left(P_{L}-P_{R}\right) f_{0}$ with the constant $f_{0}$. Apart from the soft-breaking term, these identities are similar to the supersymmetric Ward identities (1), rewritten for one-particle irreducible Green functions.

As already noted, the Slavnov-Taylor identity also describes the symmetry algebra. This information is very important in order to guarantee that the loop-corrected symmetry transformations still satisfy the (anti-)commutation relations that define supersymmetry and SU(3)-gauge invariance. We can extract information about the algebra by taking derivatives of the following kind and setting then all ghost fields and sources to zero:

$$
\begin{aligned}
0= & \frac{\delta^{3} S(\Gamma)}{\delta \epsilon \delta \bar{\epsilon} \delta Y_{j}} \\
\Rightarrow \quad 0= & \frac{\delta \Gamma}{\delta \bar{\epsilon} \delta Y_{i}} \frac{\delta \Gamma}{\delta \epsilon \delta Y_{j} \delta \varphi_{i}}+\frac{\delta \Gamma}{\delta \epsilon \delta Y_{i}} \frac{\delta \Gamma}{\delta \bar{\epsilon} \delta Y_{j} \delta \varphi_{i}} \\
& +\frac{\delta \Gamma}{\delta \epsilon \delta \bar{\epsilon} \delta Y_{j} \delta Y_{i}} \frac{\delta \Gamma}{\delta \varphi_{i}}+\frac{\delta \Gamma}{\delta \epsilon \delta \bar{\epsilon} \delta Y_{c a}} \frac{\delta \Gamma}{\delta Y_{j} \delta c_{a}} \\
& +\left.\frac{\delta^{2} s \omega^{\mu}}{\delta \epsilon \delta \bar{\epsilon}} \frac{\delta \Gamma}{\delta Y_{j} \delta \omega^{\mu}}\right|_{\mathrm{gh}=0} .
\end{aligned}
$$

The first two terms express the anticommutator of the supersymmetry transformations of $\varphi_{j}$ into $\varphi_{i}$ and of $\varphi_{i}$; the remaining terms express the right-hand side of the supersymmetry algebra

$$
\{Q, \bar{Q}\}=\text { equations of motion }+ \text { gauge transformations }
$$$$
+2 \gamma^{\mu} P_{\mu}
$$ 
where the coefficient of the translational part is fixed by

$$
\frac{\delta^{2} s \omega^{\mu}}{\delta \epsilon \delta \bar{\epsilon}}=2 \gamma^{\mu}
$$

Later we will use these identities taking further derivatives and setting all fields to zero.

\subsection{Definition of the model}

The Slavnov-Taylor identity is not the only symmetry identity in supersymmetric QCD. For an unambiguous definition of supersymmetric QCD we need four symmetry identities. We require them to be satisfied by the renormalized effective action $\Gamma$ (see [10]):

- The Slavnov-Taylor identity $S(\Gamma)=0$ expressing gauge invariance, supersymmetry and translational invariance.

- The gauge fixing condition $\frac{\delta \Gamma}{\delta B_{a}}=\frac{\delta \Gamma_{\mathrm{fix}}}{\delta B_{a}}=f_{a}+\xi B_{a}$ expressing the non-renormalization of the gauge fixing terms.

- The translational ghost equation $\frac{\delta \Gamma}{\delta \omega^{\mu}}=\frac{\delta \Gamma_{\text {ext }}}{\delta \omega^{\mu}}\left(\Gamma_{\text {ext }}\right.$ is defined in App. A) meaning that the terms involving $\omega^{\mu}$ do not receive quantum corrections.

- Global $S U(3)$ invariance and invariance under continuous $R$ transformations with the $R$-weights defined in tab. 1 1 and $C P$ invariance.

\begin{tabular}{|c||c|c|c|c|c|c|c|c|c|c|c|c|}
\hline$\varphi$ & $G_{a}^{\mu}$ & $P_{L} \tilde{g}_{a}$ & $\tilde{q}_{L}, \tilde{q}_{R}^{\dagger}$ & $q$ & $a$ & $P_{L \chi} \chi$ & $\hat{f}$ & $c_{a}$ & $P_{L} \epsilon$ & $\omega^{\nu}$ & $\bar{c}_{a}$ & $B_{a}$ \\
\hline$R$ & 0 & 1 & 1 & 0 & 0 & -1 & -2 & 0 & 1 & 0 & 0 & 0 \\
\hline$Q_{c}$ & 0 & 0 & 0 & 0 & 0 & 0 & 0 & +1 & +1 & +1 & -1 & 0 \\
\hline$G P$ & 0 & 1 & 0 & 1 & 0 & 1 & 0 & 1 & 0 & 1 & 1 & 0 \\
\hline $\operatorname{dim}$ & 1 & $3 / 2$ & 1 & $3 / 2$ & 0 & $1 / 2$ & 1 & 0 & $-1 / 2$ & -1 & 2 & 2 \\
\hline
\end{tabular}

Table 1. Quantum numbers. $R, Q_{c}, G P, \operatorname{dim}$ denote $R$-weight and ghost charge, Grassmann parity and the mass dimension, respectively. The $R$-weights of the right-handed parts $P_{R} \tilde{g}$, $P_{R} \epsilon, P_{R} \chi$ of the Majorana spinors are opposite to the ones of the left-handed parts. The quantum numbers of the external fields $Y_{i}$ can be obtained from the requirement that the products $Y_{i} s \varphi_{i}$ are neutral, bosonic and have $\operatorname{dim}=4$. The commutation rule for two general fields is $\varphi_{1} \varphi_{2}=(-1)^{G P_{1} G P_{2}} \varphi_{2} \varphi_{1}$.

\section{Determination of symmetry-restoring counterterms}

In general, regularization schemes break the defining symmetry identities. Since supersymmetric QCD is anomaly free it is always possible to restore the symmetries by adding appropriate counterterms $\Gamma_{\text {non-sym }}$ that break the symmetries by themselves. In this section we determine such counterterms at one-loop order.
A key issue in this determination is the uniqueness of the counterterms. It is not sufficient to calculate a counterterm by considering only one symmetry identity because all symmetry identities have to be satisfied simultaneously. If, however, a counterterm is determined uniquely by a certain set of symmetry identities, then it is the simultaneous solution to all identities.

The strategy in this section is the following:

- Calculate the counterterms to the supersymmetry transformations of the gluon and the gluino using identities expressing the supersymmetry and the supersymmetry algebra.

- Determine the counterterms to the supersymmetry transformations of the squarks and the quark in the same way.

- Derive the counterterm to the $\tilde{q} \tilde{g} q$ interaction using a supersymmetry identity together with the counterterms calculated before.

- Determine the counterterms to all three-particle gauge interactions and the relevant gauge transformations using identities expressing gauge invariance and the $\mathrm{SU}(3)$ algebra.

- Cross-check the result for the gauge interactions using a supersymmetry identity relating the $G_{\rho} G_{\nu} G_{\mu}$ and the $\tilde{g} \tilde{g} G_{\mu}$ interactions.

\subsection{Parametrization of the counterterms}

Counterterms can be divided into symmetric and nonsymmetric ones,

$$
\Gamma_{\mathrm{ct}}=\Gamma_{\mathrm{sym}}+\Gamma_{\text {non-sym }} .
$$

The symmetric counterterms $\Gamma_{\text {sym }}$ do not destroy any symmetry identity. They can be obtained from the classical action

$$
\Gamma_{\mathrm{cl}} \longrightarrow \Gamma_{\mathrm{cl}}+\Gamma_{\mathrm{sym}}
$$

by infinitesimal renormalization transformations for fields and parameters [9, 10]:

$$
\begin{aligned}
& G^{\mu} \rightarrow \sqrt{Z_{G}} G^{\mu}, \quad B \rightarrow{\sqrt{Z_{G}}}^{-1} B, \\
& \bar{c} \rightarrow{\sqrt{Z_{G}}}^{-1} \bar{c}, \quad \xi \rightarrow Z_{G} \xi, \\
& \tilde{g} \rightarrow \sqrt{Z_{\tilde{g}}} \tilde{g}, \quad c \rightarrow \sqrt{Z_{c}} c, \\
& P_{L, R} q \rightarrow \sqrt{Z_{q_{L, R}}} P_{L, R} q, \quad \tilde{q}_{L, R} \rightarrow \sqrt{Z_{L, R}} \tilde{q}_{L, R} \\
& Y_{i} \rightarrow{\sqrt{Z_{i}}}^{-1} Y_{i} \\
& g \rightarrow g+\delta g, \quad m_{i} \rightarrow m_{i}+\delta m_{i},
\end{aligned}
$$

where $m_{i}$ denotes all mass parameters of the theory including the soft parameters. 5 Owing to this structure the

\footnotetext{
${ }^{3}$ It is also possible to perform a matrix valued renormalization transformation of the squarks. This is important if complete on-shell renormalization conditions are desired, but for our concern the difference is not relevant.
} 
contributions of $\Gamma_{\text {sym }}$ to all self-energies and to the interaction vertex $\tilde{q} \tilde{q} G_{\mu}$ have completely arbitrary coefficients that can be chosen at will. All other contributions are functions of this choice. Formally this feature can be expressed as

$$
\Gamma_{\mathrm{sym}}=\sum_{i} \delta_{\mathrm{sym}_{i}^{(1)}}^{(1)} \mathcal{O}_{i}^{(1)}+\sum_{i} \delta_{\mathrm{sym}_{i}^{(2)}}^{(2)} \mathcal{O}_{i}^{(2)}
$$

where the operators $\mathcal{O}_{i}^{(1)}$ correspond to the self-energies and the $\tilde{q} \tilde{q} G_{\mu}$ vertex and the coefficients $\delta_{\mathrm{sym}}{ }_{i}^{(2)}$ are functions of the $\delta_{\mathrm{sym} i}^{(1)}$.

The second contribution in $\Gamma_{\mathrm{ct}}$ are the non-symmetric but symmetry-restoring counterterms $\Gamma_{\text {non-sym }}$. Since the contribution of $\Gamma_{\text {sym }}$ to the coefficients of the operators $\mathcal{O}_{i}^{(1)}$ is already completely arbitrary, it is possible to assume without loss of generality that $\Gamma_{\text {non-sym }}$ has the form

$$
\Gamma_{\text {non-sym }}=\sum_{i} \delta_{i}^{(2)} \mathcal{O}_{i}^{(2)}
$$

which means that $\Gamma_{\text {non-sym }}$ does not contain contributions to the self-energies and the $\tilde{q} \tilde{q} G_{\mu}$ vertex.

This parametrization of the counterterms we will use in the following calculations. Since it is completely general, our results are valid independently of the symmetric counterterms. Hence they hold for all renormalization schemes such as the $\overline{M S}$ or the on-shell scheme. But since this parametrization avoids redundancies it is particularly well suited for a transparent discussion. By construction, the symmetric counterterms drop from all symmetry identities, and therefore the number of unknown counterterms in the identities is minimized.

There is only one restriction we impose on $\Gamma_{\text {non-sym. }}$. Since all common regularization schemes preserve global $\mathrm{SU}(3)$ - and $C P$-invariance, we do not admit counterterms in $\Gamma_{\text {non-sym }}$ that break these symmetries.

\subsection{Gluon and gluino self energies and supersymmetry transformations}

Taking the derivative of the Slavnov-Taylor identity

$$
0=\frac{\delta^{3} S(\Gamma)}{\delta G_{b \mu} \delta \epsilon \delta \overline{\tilde{g}}_{a}}
$$

and setting all fields to zero we obtain an identity relating the gluon and gluino self energies, $]^{\text {. }}$

$$
\begin{aligned}
0= & \Gamma_{\epsilon} \overline{\tilde{g}}_{a} Y_{G_{c}^{\nu}}(-q, q) \Gamma_{G_{b \mu} G_{c \nu}}(q,-q) \\
& -\Gamma_{\tilde{g}_{c} \overline{\tilde{g}}_{a}}(q,-q) \Gamma_{G_{b \mu} \epsilon \overline{\tilde{y}_{\tilde{g}}}}(q,-q) \\
& +\frac{\delta s \chi}{\delta \epsilon} \Gamma_{G_{b \mu} \overline{\tilde{g}}_{a} \chi}(q,-q)
\end{aligned}
$$

\footnotetext{
${ }^{4}$ In the rest of this section $\Gamma$ denotes the one-loop effective action including the contributions of $\Gamma_{\text {non-sym. The symmetric }}$ counterterms do not appear since they drop from every symmetry identity.
}

In this identity the Green functions corresponding to the loop-corrected supersymmetry transformations of the gluon and the gluino are involved, and the identity determines the ratio of these two supersymmetry transformations. The last term is due to the soft supersymmetry breaking.

The notation $\Gamma_{\varphi_{1} \ldots \varphi_{n}}$ means the one-particle irreducible vertex function with external $\varphi_{1} \ldots \varphi_{n}$ fields

$$
\Gamma_{\varphi_{1} \ldots \varphi_{n}}=\left.\frac{\delta^{n} \Gamma}{\delta \varphi_{1} \ldots \delta \varphi_{n}}\right|_{\varphi_{i}=0}
$$

and the momentum arguments denote the incoming momenta (note that $\epsilon$ is a constant and thus does not carry a momentum).

In order to obtain the counterterms it is sufficient to consider the high-momentum limit. Then the softbreaking term is negligible, and the results from App. $\mathrm{Q}$ vield (the quantities $C(A)$ and $T(F)$ are defined in App. E.1, and the one-loop functions $B_{0}$ are defined in App. (D)

$$
\begin{aligned}
0= & \left(-\gamma^{\mu} q^{2}+q q^{\mu}\right) \times \\
& \left(\left[1+\delta_{Y_{G} \tilde{g} \epsilon}+\frac{\alpha_{s} C(A)}{4 \pi}\left(-B_{0}-\frac{1}{3} \theta_{\mathrm{DReg}}+B_{0}\right)\right.\right. \\
& \left.+\frac{\alpha_{s} T(F)}{4 \pi} 2 B_{0}\right] \\
& -\left[1+\delta_{G \epsilon \tilde{y} \tilde{g}}+\frac{\alpha_{s} C(A)}{4 \pi}\left(B_{0}-1 \theta_{\mathrm{DReg}}-B_{0}\right)\right. \\
& \left.\left.+\frac{\alpha_{s} T(F)}{4 \pi} 2 B_{0}\right]\right) \\
= & \left(-\gamma^{\mu} q^{2}+q q^{\mu}\right) \times \\
& \left(\delta_{Y_{G} \tilde{g} \epsilon}-\delta_{G \epsilon \tilde{y} \tilde{g}}+\frac{\alpha_{s} C(A)}{4 \pi} \frac{2}{3} \theta_{\mathrm{DReg}}\right) .
\end{aligned}
$$

Thus, in dimensional regularization $\left(\theta_{\mathrm{DReg}}=1\right)$ this identity is not satisfied on the regularized level; one has to choose non-vanishing values for the counterterms

$$
\delta_{Y_{G} \tilde{g} \epsilon}-\delta_{G \epsilon \tilde{y} \tilde{g}}=-\frac{2}{3} \frac{\alpha_{s} C(A)}{4 \pi} \theta_{\mathrm{DReg}}
$$

to the supersymmetry transformations of the gluon and the gluino.

In order to determine the individual counterterms we derive an identity corresponding to the supersymmetry algebra:

$$
\begin{aligned}
& 0=\frac{\delta^{4} S(\Gamma)}{\delta G_{b}^{\nu} \delta \epsilon \delta \bar{\epsilon} \delta Y_{G_{a}^{\mu}}^{\mu}}
\end{aligned}
$$

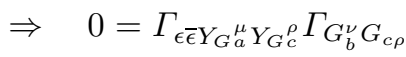

$$
\begin{aligned}
& +\Gamma_{Y_{G}^{\mu} \tilde{g}_{c} \bar{\epsilon}} \Gamma_{G_{b}^{\nu} \epsilon \overline{\bar{y}} \bar{g} c}-\Gamma_{G_{b}^{\nu} \tilde{y}_{\tilde{g} c} \bar{\epsilon}} \Gamma_{Y_{G}{ }_{a}^{\mu} \in \bar{g}_{c}} \\
& +\Gamma_{G_{b}^{\nu} \epsilon \bar{\epsilon} Y_{c c}} \Gamma_{Y_{G a}^{\mu} c_{c}}
\end{aligned}
$$

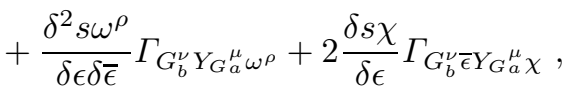


which yields in the high-momentum limit

$$
\begin{aligned}
0= & -2\left(q_{\mu} \gamma_{\nu}-\not d g_{\mu \nu}\right) \delta_{a b}\left(1+\delta_{G \epsilon \tilde{y}_{\tilde{g}}}+\delta_{Y_{G} \tilde{g} \epsilon}\right) \\
& +2\left(q_{\mu} \gamma_{\nu}\right) \delta_{a b}\left(1+\delta_{c Y_{G}}+\delta_{G \epsilon \bar{\epsilon} Y_{c}}\right) \\
& -2 \not q g_{\mu \nu} \delta_{a b} .
\end{aligned}
$$

The physical meaning of this identity is the constraint of the supersymmetry algebra on the product of the transformations of the gluon into the gluino and backwards correspondingly it determines the sum of the two counterterms. In contrast, the previous identity (22) determines the ratio of the two supersymmetry transformations and therefore the difference of the counterterms.

Hence, taken together both identities lead to a unique value for the counterterms

$$
\begin{aligned}
\delta_{Y_{G} \tilde{g} \epsilon} & =-\delta_{G \epsilon \tilde{y} \tilde{g}}=-\frac{\alpha_{s} C(A)}{4 \pi} \frac{1}{3} \theta_{\mathrm{DReg}}, \\
\delta_{G \epsilon \bar{\epsilon} Y_{c}} & =-\delta_{c Y_{G}} .
\end{aligned}
$$

The counterterms $\delta_{G \epsilon \bar{\epsilon} Y_{c}}$ and $\delta_{c Y_{G}}$ we will not need in the following.

This result is a simple illustration of the discussion at the beginning of this section. Apparently there are infinitely many different counterterms that solve (25) or equivalently (22), i.e. restore the gluon-gluino identity. Only one particular choice, however, also solves the second identity (26). Therefore it is essential to take into account the identities that correspond to the supersymmetry algebra.

\subsection{Quark and squark self energies and supersymmetry transformations}

Using the following derivative of the Slavnov-Taylor identity we obtain an identity relating the quark and squark self energies:[]

$$
\begin{aligned}
0= & \frac{\delta^{3} S(\Gamma)}{\delta q \delta \tilde{q}_{i}^{\dagger} \delta \bar{\epsilon}} \\
\Rightarrow \quad 0= & \sum_{j=1,2} \Gamma_{q \bar{\epsilon} \tilde{y}_{j}} \Gamma_{\tilde{q}_{i}^{\dagger} \tilde{q}_{j}}-\Gamma_{\tilde{q}_{i}^{\dagger} y \bar{\epsilon}} \Gamma_{q \bar{q}} \\
& +\frac{\delta s \bar{\chi}}{\delta \bar{\epsilon}} \Gamma_{q \tilde{q}_{i}^{\dagger} \bar{\chi}} .
\end{aligned}
$$

In the high-momentum limit this reduces to (the quantity $C(F)$ is defined in App. E.1)

$$
\begin{aligned}
0= & \sqrt{2} q^{2}\left(S_{i L} P_{L}-S_{i R} P_{R}\right)\left(1+\delta_{\tilde{y} \epsilon q}+\frac{\alpha_{s} C(F)}{4 \pi} B_{0}\right) \\
& -\sqrt{2} q^{2}\left(S_{i L} P_{L}-S_{i R} P_{R}\right) \times \\
& \left(1+\delta_{y \tilde{q} \epsilon}+\frac{\alpha_{s} C(F)}{4 \pi}\left(2 B_{0}-1 \theta_{\mathrm{DReg}}-\left(B_{0}\right)\right)\right),(31)
\end{aligned}
$$

\footnotetext{
${ }^{5}$ We suppress the colour indices since the following identities are trivial in colour space.
}

which is satisfied provided the counterterms fulfil

$$
\delta_{\tilde{y} \epsilon q}-\delta_{y \tilde{q} \epsilon}=-\frac{\alpha_{s} C(F)}{4 \pi} \theta_{\mathrm{DReg}} .
$$

Again, these are counterterms to the supersymmetry transformations, and the considered identity only fixes their difference.

As in the gluon/gluino case we need an additional identity corresponding to the supersymmetry algebra to find unique values for these counterterms, given by

$$
\begin{aligned}
0= & \frac{\delta^{4} S(\Gamma)}{\delta \tilde{q}_{j} \delta \epsilon \delta \bar{\epsilon} \delta \tilde{y}_{i}} \\
\Rightarrow \quad 0= & -\Gamma_{\epsilon \bar{\epsilon} \tilde{y}_{i} \tilde{y}_{k}^{\dagger}} \Gamma_{\tilde{q}_{j} \tilde{q}_{k}^{\dagger}} \\
& +\Gamma_{\tilde{y}_{i} q \bar{\epsilon}} \Gamma_{\tilde{q}_{j} \epsilon \bar{y}}-\Gamma_{\tilde{q}_{j} y^{C} \bar{\epsilon}} \Gamma_{\tilde{y}_{i} \epsilon \bar{q}^{C}}+\frac{\delta^{2} s \omega^{\rho}}{\delta \epsilon \delta \bar{\epsilon}} \Gamma_{\tilde{q}_{j} \tilde{y}_{i} \omega^{\rho}} \\
& +\frac{\delta s \chi}{\delta \epsilon} \Gamma_{\tilde{q}_{j} \epsilon \tilde{y}_{i} \chi}+\frac{\delta s \chi}{\delta \bar{\epsilon}} \Gamma_{\tilde{q}_{j} \epsilon \tilde{y}_{i} \chi} .
\end{aligned}
$$

In the high-momentum limit only the second, third and fourth term contribute, with the result

$$
0=2 \not \phi \delta_{i j}\left(1+\delta_{\tilde{y} \epsilon q}+\delta_{y \tilde{q} \epsilon}\right)-2 \not \phi \delta_{i j} .
$$

The unique solution for the counterterms is thus

$$
\delta_{\tilde{y} \epsilon q}=-\delta_{y \tilde{q} \epsilon}=-\frac{\alpha_{s} C(F)}{4 \pi} \frac{1}{2} \theta_{\mathrm{DReg}} .
$$

\subsection{Gluino-Quark-Squark vertex}

One very important consequence of supersymmetry is the relation between the interactions of quarks and squarks with gluons and gluinos. This relation can be expressed by the following identity:

$$
\begin{aligned}
0= & \frac{\delta^{4} S(\Gamma)}{\delta \tilde{q}_{L} \delta \tilde{q}_{L}^{\dagger} \delta \tilde{g}_{a R} \delta \bar{\epsilon}} \\
\Rightarrow \quad 0= & \Gamma_{\tilde{g}_{a R} \bar{\epsilon} Y_{G}{ }_{c}^{\mu}}(k,-k) \Gamma_{\tilde{q}_{L} \tilde{q}_{L}^{\dagger} G_{c \mu}}\left(p,-p^{\prime}, k\right) \\
& -\Gamma_{\tilde{q}_{L} \tilde{q}_{L}^{\dagger} \tilde{y}_{\tilde{g} \overline{ }} \bar{\epsilon}}\left(p,-p^{\prime}, k\right) \Gamma_{\tilde{g}_{a R} \bar{g}_{c}}(k,-k) \\
& +\sum_{j=1,2}\left(\Gamma_{\tilde{g}_{a R} \bar{\epsilon} \tilde{q}_{L} \tilde{y}_{j}}\left(k, p,-p^{\prime}\right) \Gamma_{\tilde{q}_{L}^{\dagger} \tilde{q}_{j}}\left(-p^{\prime}, p^{\prime}\right)\right. \\
& \left.-\Gamma_{\tilde{g}_{a R} \bar{\epsilon} \tilde{q}_{L}^{\dagger} \tilde{y}_{j}^{\dagger}}\left(k,-p^{\prime}, p\right) \Gamma_{\tilde{q}_{L} \tilde{q}_{j}^{\dagger}}(p,-p)\right) \\
& -\Gamma_{\tilde{q}_{L}^{\dagger} y \bar{\epsilon}}\left(-p^{\prime}, p^{\prime}\right) \Gamma_{\tilde{q}_{L}} \tilde{g}_{a R} \bar{q} \\
& +\Gamma_{\tilde{q}_{L}^{\dagger} \tilde{g}_{a R} q}\left(-p^{\prime}, k, p\right) \Gamma_{\tilde{q}_{L} \bar{\epsilon} \bar{y}}(p,-p) \\
& +\frac{\delta s \bar{\chi}}{\delta \bar{\epsilon}} \Gamma_{\tilde{q}_{L} \tilde{q}_{L}^{\dagger} \tilde{g}_{a R} \bar{\chi}^{\prime}\left(p,-p^{\prime}, k, 0\right) .}
\end{aligned}
$$

In this identity we choose definite interaction eigenstates for the squarks as external legs and consider only the righthanded part of the gluino $\tilde{g}_{R}=P_{R} \tilde{g}$. This simplifies the

\footnotetext{
${ }^{6}$ The values for the vertex functions involving $y^{C}, \bar{q}^{C}$ can easily be inferred from the corresponding ones involving $\bar{y}, q$ using the flipping rules of [14].
} 
computation, but due to (only softly broken) $C$ - and $P$ invariance it is sufficient to obtain the supersymmetryrestoring counterterms to all $\tilde{q} \tilde{g} q$-interactions.

The identity (36) has to hold for arbitrary external momenta. Since the counterterms to the interactions are momentum-independent, it is very convenient to consider the limit $m_{i} \ll\left|k_{\mu}\right| \ll\left|p_{\mu}\right|=\left|\left(p^{\prime}-k\right)_{\mu}\right|$, where $m_{i}$ denote the masses in the theory. In this limit all masses can be neglected, and $k$ can be neglected compared to $p$ except in the terms that would lead to infrared divergences for $k=0$. The only remaining terms are

$$
\begin{aligned}
0= & \Gamma_{\tilde{g}_{a R} \bar{\epsilon} Y_{G c}^{\mu}}(k,-k) \Gamma_{\tilde{q}_{L} \tilde{q}_{L}^{\dagger} G_{c \mu}}\left(p,-p^{\prime}, k\right) \\
& +\sum_{j=1,2}\left(\Gamma_{\tilde{g}_{a R} \bar{\epsilon} \tilde{q}_{L} \tilde{y}_{j}}\left(k, p,-p^{\prime}\right) \Gamma_{\tilde{q}_{L}^{\dagger} \tilde{q}_{j}}\left(-p^{\prime}, p^{\prime}\right)\right. \\
& \left.-\Gamma_{\tilde{g}_{a R} \bar{\epsilon} \tilde{q}_{L}^{\dagger} \tilde{y}_{j}^{\dagger}}\left(k,-p^{\prime}, p\right) \Gamma_{\tilde{q}_{L} \tilde{q}_{j}^{\dagger}}(p,-p)\right) \\
& -\Gamma_{\tilde{q}_{L}^{\dagger} y \bar{\epsilon}}\left(-p^{\prime}, p^{\prime}\right) \Gamma_{\tilde{q}_{L} \tilde{g}_{a R} \bar{q}}\left(p, k,-p^{\prime}\right) .
\end{aligned}
$$

The physical meaning of the first and the last term is obvious. They relate the $\tilde{q} \tilde{q} G_{\mu}$ and $\tilde{q} q \tilde{g}$ interactions, multiplied with the corresponding supersymmetry transformations of the gluon and quark, respectively. The other terms are particularly interesting: They involve supersymmetry transformations of squarks into products of the form $\bar{\epsilon} \tilde{g} \tilde{q}$ - such transformations are not present at the tree level but can be generated at one-loop order. The corresponding Feynman graphs are all finite and thus involve no regularization ambiguity. In the limit defined above the results are

$$
\begin{aligned}
& 0=-2 \not p g P_{R} T^{a}\left(1+\delta_{Y_{G} \tilde{g} \epsilon}\right. \\
&\left.+\frac{\alpha_{s}}{4 \pi} C(A)\left[B_{0}\left(k^{2}\right)+B_{0}+\frac{3}{2} p^{2} C_{1}\right]\right) \\
&+\not p g P_{R} T^{a} p^{2} \frac{\alpha_{s}}{4 \pi}\left(\frac{C(A)}{2}\left[2 C_{0}+C_{1}\right]\right. \\
&\left.+\frac{C(A)}{2}\left[2 C_{0}-C_{1}\right]+C(F)\left[2 C_{1}\right]\right) \\
&+2 \not p g P_{R} T^{a}\left(1+\delta_{y \tilde{q} \epsilon}+\delta_{\tilde{q} \tilde{q} q}\right. \\
&+\frac{\alpha_{s}}{4 \pi}\left[C(F)\left(-B_{0}+B_{0}-p^{2} C_{1}\right)\right. \\
&\left.\left.+\frac{C(A)}{2}\left(4 B_{0}+7 p^{2} C_{1}-2 \theta_{\mathrm{DReg}}\right)\right]\right)
\end{aligned}
$$

The arguments of the one-loop functions are as in eq. (67), except where indicated differently. Most of the terms cancel, leaving only

$$
\begin{array}{r}
0=2 \not p g \\
P_{R} T^{a}\left(-\delta_{Y_{G} \tilde{g} \epsilon}+\frac{\alpha_{s}}{4 \pi} C(A)\left[p^{2} C_{0}-B_{0}\left(k^{2}\right)\right.\right. \\
\left.\left.+2 p^{2} C_{1}+B_{0}-1 \theta_{\mathrm{DReg}}\right]+\delta_{y \tilde{q} \epsilon}+\delta_{\tilde{q} \tilde{g} q}\right)
\end{array}
$$

For the terms $C_{0}$ and $B_{0}\left(k^{2}\right)$, which are infrared divergent for $\left|\frac{k}{p}\right| \rightarrow 0$, one can easily verify the identity

$$
\begin{array}{r}
\lim _{k \rightarrow 0}\left(p^{2} C_{0}\left(p^{2},(p+k)^{2}, k^{2}, 0,0,0\right)-B_{0}\left(k^{2}, 0,0\right)\right)= \\
-2 p^{2} C_{1}-B_{0} .
\end{array}
$$

Thus, all $B_{0}, C_{i}$ functions cancel perfectly, leaving an identity for the counterterms,

$$
0=\delta_{\tilde{q} \tilde{g} q}-\delta_{Y_{G} \tilde{g} \epsilon}-\frac{\alpha_{s}}{4 \pi} C(A) \theta_{\mathrm{DReg}}+\delta_{y \tilde{q} \epsilon}
$$

that has a unique solution for the counterterm to the $\tilde{q} \tilde{g} q$ interaction

$$
\delta_{\tilde{q} \tilde{g} q}=\frac{\alpha_{s}}{4 \pi}\left(\frac{2}{3} C(A)-\frac{1}{2} C(F)\right) \theta_{\mathrm{DReg}}
$$

or written in terms of a counterterm Lagrangian

$$
\begin{aligned}
& \mathcal{L}_{\text {non-sym, } \tilde{q} \tilde{g} q}=-\delta_{\tilde{q} \tilde{g} q} \sqrt{2} g \times \\
& \quad\left(\tilde{q}_{L}^{\dagger} \bar{g} P_{L} q+\bar{q} P_{R} \tilde{g} \tilde{q}_{L}-\tilde{q}_{R}^{\dagger} \overline{\tilde{g}} P_{R} q-\bar{q} P_{L} \tilde{g} \tilde{q}_{R}\right) .
\end{aligned}
$$

Here the result obtained for the $\bar{q} P_{R} \tilde{g} \tilde{q}_{L}$-interaction has been extended to the other $\tilde{q} \tilde{g} q$-interactions. The respective calculations can be done in the same way and yield the same result due to hermiticity and (softly broken) $C$ - and $P$-invariance. As can be easily checked, $R$-invariance is not violated by the regularization, and therefore $R$-violating counterterms like $\bar{q} P_{R} \tilde{g} \tilde{q}_{R}$ are not necessary.

Hence, in dimensional regularization $\left(\theta_{\text {DReg }}=1\right)$ an additional counterterm is necessary to compensate the supersymmetry breaking of the regularization. In dimensional reduction, however, this counterterm is not necessary. Both results have already been anticipated in 11], so eq. (42) provides a confirmation on the basis of a rigorous definition of the model using the Slavnov-Taylor identity.

We want to stress that the result for the counterterm $\delta_{\tilde{q} \tilde{g} q}$ is unique. The uniqueness guarantees that $\delta_{\tilde{q} \tilde{g} q}$ is not only the solution of (36) but of all symmetry identities. To arrive at this result the unambiguous calculation of the counterterms to the supersymmetry transformations in the preceding subsections has been necessary. In particular, in dimensional reduction only the combination of all these calculations implies that supersymmetry is preserved in this sector.

\subsection{Gauge interactions}

In the previous subsection we have determined the counterterm to the $\tilde{q} \tilde{g} q$ interaction. All other trilinear interactions of supersymmetric QCD are gauge interactions: $\tilde{q} \tilde{q} G_{\mu}, q q G_{\mu}, \tilde{g} \tilde{g} G_{\mu}$, and $G_{\rho} G_{\nu} G_{\mu}$. We have explicitely checked that all symmetry identities that determine these interactions hold automatically in both regularization schemes, although $\gamma_{5}$-interactions are involved. Since these identities are not due to supersymmetry, and since the gauge invariance of both schemes is generally known, we are brief in this subsection and restrict ourselves to a sketch of the calculations.

The identities that determine the counterterms to the gauge interactions can be obtained from the following 
derivatives of the Slavnov-Taylor identity:

$$
\begin{array}{cc}
\frac{\delta^{3} S(\Gamma)}{\delta \tilde{q}^{\dagger} \delta \tilde{q} \delta c_{a}}, & \frac{\delta^{4} S(\Gamma)}{\delta \tilde{q} \delta c_{a} \delta c_{b} \delta \tilde{y}}, \\
\frac{\delta^{3} S(\Gamma)}{\delta q \delta \bar{q} \delta c_{a}}, & \frac{\delta^{4} S(\Gamma)}{\delta q \delta c_{a} \delta c_{b} \delta \bar{y}} \\
\frac{\delta^{3} S(\Gamma)}{\delta \tilde{g}_{c} \delta \overline{\tilde{g}}_{d} \delta c_{a}}, & \frac{\delta^{4} S(\Gamma)}{\delta \tilde{g}_{c} \delta c_{a} \delta c_{b} \delta \overline{\tilde{y}_{\tilde{g}}} d} \\
\frac{\delta^{3} S(\Gamma)}{\delta G_{c}^{\nu} \delta G_{d}^{\mu} \delta c_{a}}, & \frac{\delta^{4} S(\Gamma)}{\delta c_{a} \delta c_{b} \delta Y_{G_{d}}^{\mu}}
\end{array}
$$

The first set of these identities expresses the gauge invariance, whereas the second set corresponds to the symmetry algebra. Owing to our parametrization (see sec. 3.1) there is no symmetry-violating counterterm to the $\tilde{q} \tilde{q} G_{\mu}$ interaction. Rather, this interaction defines the gauge coupling and determines the other counterterms. I Evaluating these identities explicitely at one-loop order we find that they are satisfied at the regularized level, which has the following consequence for the counterterms: 8

$$
\begin{aligned}
\delta_{c Y_{G}} & =\delta_{\tilde{q} c \tilde{y}}, & \delta_{\tilde{q} c \tilde{y}} & =\delta_{c c Y_{c}}, \\
\delta_{c Y_{G}}+\delta_{q q G} & =\delta_{q c y}, & \delta_{q c y} & =\delta_{c c Y_{c}}, \\
\delta_{c Y_{G}}+\delta_{\tilde{g} \tilde{g} G} & =\delta_{\tilde{g} c \tilde{y} \tilde{g}}, & \delta_{\tilde{g} c \tilde{y} \tilde{g}} & =\delta_{c c Y_{c}}, \\
\delta_{c Y_{G}}+\delta_{G G G} & =\delta_{c Y_{G} G}, & \delta_{c Y_{G} G} & =\delta_{c c Y_{c}} .
\end{aligned}
$$

These identities have the unique solution

$$
\begin{gathered}
\delta_{q q G}=\delta_{\tilde{g} \tilde{g} G}=\delta_{G G G}=0, \\
\delta_{\tilde{q} c \tilde{y}}=\delta_{q c y}=\delta_{\tilde{g} c \tilde{y} \tilde{g}}=\delta_{c Y_{G} G}=\delta_{c Y_{G}},
\end{gathered}
$$

where the only freedom is the value of $\delta_{c Y_{G}}$, which is related to the residue of the ghost propagator and can be fixed by specifying a renormalization condition for the latter. The important result, however, is that the nonsymmetric counterterms to all gauge interactions turn out to be zero. To obtain this result, it has been essential to verify in particular the second set of identities in eq. (44), corresponding to the $\mathrm{SU}(3)$-algebra.

\subsection{Gluon-Gluino-Gluino vertex}

In the previous subsection the result

$$
\delta_{\tilde{g} \tilde{g} G}=\delta_{G G G}=0
$$

\footnotetext{
7 There is nothing special about this interaction; we could have chosen any other gauge interaction instead to define the gauge coupling.

${ }^{8}$ In particular we find the - already non-trivial - result that the Lorentz- and SU(3)-structure of the counterterms must be identical to the one of the tree-level interactions. For instance, in general there could be two linearly independent counterterms to the $G G G$ interaction
}

$$
\begin{aligned}
\mathcal{L}_{\text {non-sym } G G G}= & \delta_{G G G} \frac{1}{2} f_{a b c} G_{a}^{\mu} G_{b}^{\nu} \partial_{\mu} G_{c \nu} \\
& +\delta_{G G G 2} \operatorname{Tr}\left(G^{\mu} G_{\mu} \partial^{\nu} G_{\nu}\right),
\end{aligned}
$$

but only $\delta_{G G G}$ can contribute. was derived using gauge invariance. On the other hand, the $\tilde{g} \tilde{g} G_{\mu}$ and $G_{\rho} G_{\nu} G_{\mu}$ interactions are also related by supersymmetry. Consistency requires that the relation imposed by supersymmetry must be automatically satisfied, which gives an important check. Equivalently, if we use supersymmetry to rederive the counterterms we must obtain a result compatible with (47).

The following identity, which is due to supersymmetry, connects the triple-gluon vertex and the gluon-gluinogluino vertex:

$$
\begin{aligned}
& 0=\frac{\delta^{4} S(\Gamma)}{\delta G_{a}^{\mu} \delta G_{b}^{\nu} \delta \tilde{g}_{c} \delta \bar{\epsilon}} \\
& \Rightarrow \quad 0=\left[\Gamma_{G_{b}^{\nu} \tilde{g}_{c} \bar{\epsilon} Y_{G_{d}^{\rho}}^{\rho}} \Gamma_{G_{a}^{\mu} G_{d \rho}}+((\mu, a) \leftrightarrow(\nu, b))\right]
\end{aligned}
$$

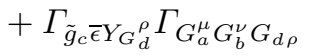

$$
\begin{aligned}
& -\left[\Gamma_{G_{b}^{\nu} \tilde{y} \tilde{g} d \bar{\epsilon}} \Gamma_{G_{a}^{\mu} \tilde{g}_{c} \overline{\tilde{g}}_{d}}+((\mu, a) \leftrightarrow(\nu, b))\right]
\end{aligned}
$$

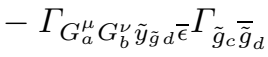

$$
\begin{aligned}
& +\frac{\delta s \bar{\chi}}{\delta \bar{\epsilon}} \Gamma_{G_{a}^{\mu} G_{b}^{\nu} \tilde{g}_{c} \bar{\chi}} .
\end{aligned}
$$

The vertex functions involving the $\epsilon$ ghost correspond to supersymmetry transformations.

The identity (48) has to hold for arbitrary external momenta, but for the purpose of deriving counterterms it is sufficient to look at the limit $m_{i} \ll\left|p_{b}\right| \ll\left|p_{a}\right|$ and to consider only the leading terms in $p_{a}$. In this limit the soft-breaking term and all the masses do not contribute, and all contributions get a simple analytical form, and all non-local terms cancel:

$$
\begin{aligned}
0= & i g f_{a b c}\left(\left(\frac{2 C(A)}{3} \frac{\alpha_{s}}{4 \pi} \theta_{\mathrm{DReg}}\right.\right. \\
& \left.+\delta_{G G G}+\delta_{Y_{G} \tilde{g} \epsilon}-\delta_{\tilde{y}_{\tilde{g}} \epsilon G G}\right) \not_{a} g_{\mu \nu} \\
& +\left(-\delta_{G \tilde{g} \tilde{g}}-\delta_{G \epsilon \tilde{y} \tilde{g}}+\delta_{\tilde{y}_{\tilde{g}} \epsilon G G}\right) \gamma_{\mu} \gamma_{\nu} p_{a} \\
& +\left(\frac{2 C(A)}{3} \frac{\alpha_{s}}{4 \pi} \theta_{\mathrm{DReg}}-\delta_{G \tilde{g} \tilde{g}}+\delta_{G G G}\right. \\
& \left.\left.-\delta_{G \epsilon \tilde{y} \tilde{g}}+\delta_{Y_{G} \tilde{g} \epsilon}\right)\left(\gamma_{\nu} p_{a \mu}-2 \gamma_{\mu} p_{a \nu}\right)\right) .
\end{aligned}
$$

From the longitudinal part of this equation, which is obtained by the contraction with $p_{a}^{\mu}$, we get

$$
\delta_{\tilde{y}_{\tilde{g}} \epsilon G}=\frac{2 C(A)}{3} \frac{\alpha_{s}}{4 \pi} \theta_{\mathrm{DReg}}+\delta_{G G G}+\delta_{Y_{G} \tilde{g} \epsilon} .
$$

This result fixes the counterterm for the vertex function $\Gamma_{G_{a}^{\mu} G_{b}^{\nu} \tilde{y} \tilde{g} d \bar{\epsilon}}$ in terms of the other counterterms. Inserting it in (49) yields

$$
\delta_{G \tilde{g} \tilde{g}}=\frac{2 C(A)}{3} \frac{\alpha_{s}}{4 \pi} \theta_{\mathrm{DReg}}+\delta_{G G G}-\delta_{G \epsilon \tilde{y} \tilde{g}}+\delta_{Y_{G} \tilde{g} \epsilon} .
$$

This is an expression for the counterterm of the gluongluino-gluino interaction in terms of the counterterm of the triple-gluon vertex and the counterterms $\delta_{G \epsilon \tilde{y}_{\tilde{g}}}$ and 
$\delta_{Y_{G} \tilde{g} \epsilon}$, calculated in subsec. 3.2. The results for $\delta_{G \epsilon \tilde{y} \tilde{g}}$ and $\delta_{Y_{G} \tilde{g} \epsilon}$ show that these counterterms cancel the first term on the r.h.s. of eq. (51), thus yielding

$$
\delta_{G \tilde{g} \tilde{g}}=\delta_{G G G},
$$

in agreement with eq. (47).

\section{Conclusions}

We have calculated symmetry-restoring counterterms in supersymmetric QCD. We have found that in DRed no non-symmetric counterterms are necessary at the one-loop level, neither for the trilinear interactions nor for the symmetry transformation operators. While identities like eq. (25) have been checked in the literature [3], the result that the loop-corrected symmetry transformations automatically satisfy the right algebra is new. In DReg counterterms for the $\tilde{q} \tilde{g} q$-interaction and for most symmetry transformations are required.

In order to obtain unique results we have had to take into account loop corrections to the symmetry transformations and to renormalize them in such a way that the SU(3)- and the supersymmetry algebra are satisfied. The fact that the counterterms to these symmetry transformations can be unambiguously calculated is the main advantage of the Slavnov-Taylor identity compared to simple supersymmetric Ward identities like eq. (11).

The uniqueness of our results guarantees that the counterterms derived from only few symmetry identities remain correct even if we take into account all symmetry identities simultaneously. Only to the vertex functions we have not considered additional counterterms may be required. Therefore our results for DRed, where no counterterm is needed, constitute a definite test of the supersymmetry of the scheme for the considered cases. And when the use of dimensional regularization is desirable, for instance because the standard $\overline{M S}$ mass-factorization scheme should be used [12], our results show how to establish all symmetries.

Let us now give some final remarks on the necessity to calculate loop corrections and counterterms to the symmetry transformations. This necessity might seem disturbing. But using the Feynman rules and the explicit form of the Slavnov-Taylor identity we have provided, the calculations turn out to be straightforward. In fact, the one-loop corrections to the symmetry transformations are much simpler than the one-loop corrections to the interaction vertices we have considered.

As mentioned in [8] the appearance of loop corrections to the symmetry transformations can be traced back to two reasons. First, the non-linearity of the BRS transformations can cause a difference between expectation values $\langle s \varphi\rangle$ and the respective products of the classical fields. In the loop diagrams the non-linearity is the reason for the triple- or quartic couplings to the external $Y$ sources. A second reason is the supersymmetry breaking of the gauge fixing, which necessitates compensating terms involving the supersymmetry ghosts. The corresponding Feynman rules appear in most of the loop diagrams to the supersymmetry transformations. Owing to these terms, loop corrections are even possible to supersymmetry transformations that are linear at the tree level, e.g. the one of the gluon (see fig. 2).

This sheds a light on the deep connection of gauge invariance and supersymmetry, which was a major complication in the renormalization for a long time and has enforced the introduction of the Slavnov-Taylor identity. Our results help to get a quantitative understanding of such general properties of the theory.

We thank E. Kraus for helpful comments and T. Fritzsche, T. Hahn, and C. Schappacher for useful discussions and valuable advice in the use of the Mathematica packages FeynArts and Form Calc.

\section{A Lagrangian and BRS transformations}

In this section we give the explicit form of the BRS transformations and the Lagrangian of supersymmetric QCD as a specialized version of the general Yang-Mills theories discussed in 10. In contrast to there, we use 4-spinors throughout in order to obtain Feynman rules that can be used in a straightforward way.

\section{A.1 BRS Transformations}

We combine gauge and supersymmetry transformations and translations in a single anticommuting BRS operator $s$. On the "physical" fields (i.e. the ones carrying no ghost number) $s$ acts as the sum of gauge and supersymmetry transformations and translations, where the transformation parameters have been promoted to ghost fields $c_{a}(x), \epsilon, \omega^{\mu}$ :

$$
\begin{aligned}
s G^{\mu}= & \partial^{\mu} c-i g\left[c, G^{\mu}\right]+\bar{\epsilon} \gamma^{\mu} \tilde{g}-i \omega^{\nu} \partial_{\nu} G^{\mu}, \\
s \tilde{g}= & -i g\{c, \tilde{g}\}-\frac{1}{2} \sigma^{\rho \sigma} \epsilon F_{\rho \sigma}+D\left(P_{L}-P_{R}\right) \epsilon-i \omega^{\nu} \partial_{\nu} \tilde{g} \\
s \overline{\tilde{g}}= & -i g\{c, \overline{\tilde{g}}\}+\frac{1}{2} \bar{\epsilon} \sigma^{\rho \sigma} F_{\rho \sigma}+\bar{\epsilon}\left(P_{L}-P_{R}\right) D-i \omega^{\nu} \partial_{\nu} \overline{\tilde{g}} \\
s \tilde{q}_{L}= & -i g c \tilde{q}_{L}+\sqrt{2} \bar{\epsilon} P_{L} q-i \omega^{\nu} \partial_{\nu} \tilde{q}_{L} \\
s \tilde{q}_{L}^{\dagger}= & +i g \tilde{q}_{L}^{\dagger} c+\sqrt{2} \bar{q} P_{R} \epsilon-i \omega^{\nu} \partial_{\nu} \tilde{q}_{L}^{\dagger} \\
s \tilde{q}_{R}= & -i g c \tilde{q}_{R}-\sqrt{2} \bar{\epsilon} P_{R} q-i \omega^{\nu} \partial_{\nu} \tilde{q}_{R} \\
s \tilde{q}_{R}^{\dagger}= & +i g \tilde{q}_{R}^{\dagger} c-\sqrt{2} \bar{q} P_{L} \epsilon-i \omega^{\nu} \partial_{\nu} \tilde{q}_{R}^{\dagger}, \\
s q= & -i g c q+\sqrt{2} m\left(\tilde{q}_{L} P_{R}-\tilde{q}_{R} P_{L}\right) \epsilon \\
& +\sqrt{2} i D_{\mu}\left(\tilde{q}_{L} P_{L}-\tilde{q}_{R} P_{R}\right) \gamma^{\mu} \epsilon-i \omega^{\nu} \partial_{\nu} q \\
s \bar{q}= & -i g \bar{q} c+\sqrt{2} m \bar{\epsilon}\left(-\tilde{q}_{L}^{\dagger} P_{L}+\tilde{q}_{R}^{\dagger} P_{R}\right) \\
& +\sqrt{2} i \bar{\epsilon} \gamma^{\mu}\left(\left(D_{\mu} \tilde{q}_{L}\right)^{\dagger} P_{R}-\left(D_{\mu} \tilde{q}_{R}\right)^{\dagger} P_{L}\right)-i \omega^{\nu} \partial_{\nu} \bar{q} \\
s a= & \sqrt{2} \bar{\epsilon} P_{L} \chi-i \omega^{\nu} \partial_{\nu} a \\
s a^{\dagger}= & \sqrt{2} \bar{\chi} P_{R} \epsilon-i \omega^{\nu} \partial_{\nu} a^{\dagger}, \\
s \chi= & \sqrt{2}\left(P_{L} \hat{f}-P_{R} \hat{f}^{\dagger}\right) \epsilon \\
& +\sqrt{2} i\left(P_{L} \partial_{\mu} a-P_{R} \partial_{\mu} a^{\dagger}\right) \gamma^{\mu} \epsilon-i \omega^{\nu} \partial_{\nu} \chi \\
s f= & \sqrt{2} i \bar{\epsilon} \gamma^{\mu} \partial_{\mu} P_{L} \chi-i \omega^{\nu} \partial_{\nu} f \\
s f^{\dagger}= & -\sqrt{2} i \partial_{\mu} \bar{\chi} \gamma^{\mu} P_{L} \epsilon-i \omega^{\nu} \partial_{\nu} f^{\dagger} .
\end{aligned}
$$


Here we have used the notation $G^{\mu}=G_{a}^{\mu} T^{a}$ etc. for all fields in the adjoint representation of the gauge group. Furthermore, we have used the gauge covariant derivative

$$
D^{\mu}=\partial^{\mu}+i g T^{a} G_{a}^{\mu}
$$

where $T^{a}$ has to be replaced by $-i f_{a b c}$ in the adjoint representation, the field strength tensor

$$
F_{a}^{\mu \nu}=\partial^{\mu} G_{a}^{\nu}-\partial^{\nu} G_{a}^{\mu}-g f_{a b c} G_{b}^{\mu} G_{c}^{\nu}
$$

and the abbreviation

$$
D_{a}=-g\left(\tilde{q}_{L}^{\dagger} T^{a} \tilde{q}_{L}-\tilde{q}_{R}^{\dagger} T^{a} \tilde{q}_{R}\right) .
$$

Generally, the BRS operator has the important nilpotency property

$$
s^{2}=0+\text { field equations }
$$

provided the statistics of the ghost fields is "wrong", i.e. opposite to the one required by the spin-statistics theorem, and the BRS transformations of the ghosts themselves are given by the structure constants of the symmetry algebra, as follows:

$$
\begin{aligned}
s c & =-i g c^{2}+i \bar{\epsilon} \gamma^{\mu} \epsilon G_{\mu}-i \omega^{\nu} \partial_{\nu} c, \\
s \epsilon & =0 \\
s \omega^{\nu} & =\bar{\epsilon} \gamma^{\nu} \epsilon .
\end{aligned}
$$

Finally, for gauge fixing we introduce Faddeev-Popov antighosts $\bar{c}_{a}$ and auxiliary fields $B_{a}$ with BRS transformations

$$
\begin{aligned}
s \bar{c} & =B-i \omega^{\nu} \partial_{\nu} \bar{c}, \\
s B & =i \bar{\epsilon} \gamma^{\nu} \epsilon \partial_{\nu} \bar{c}-i \omega^{\nu} \partial_{\nu} B .
\end{aligned}
$$

\section{A.2 Lagrangian}

The Lagrangian of supersymmetric QCD consists of one part containing the physical fields only, and one part containing the ghosts and the external fields. The first part is given by

$$
\begin{aligned}
& \mathcal{L}_{\text {phys }}=\mathcal{L}_{\text {kin }}+\mathcal{L}_{m}+\mathcal{L}_{\text {soft }}, \\
& \mathcal{L}_{\text {kin }}=-\frac{1}{4}\left(F_{\mu \nu}^{a}\right)^{2}+\frac{1}{2} \overline{\tilde{g}}_{a} i \gamma^{\mu} D_{\mu} \tilde{g}_{a} \\
& +\bar{q} i \gamma^{\mu} D_{\mu} q+\left|D^{\mu} \tilde{q}_{L}\right|^{2}+\left|D^{\mu} \tilde{q}_{R}\right|^{2}-\frac{1}{2} D_{a} D_{a} \\
& -\sqrt{2} g\left(\tilde{q}_{L}^{\dagger} \overline{\tilde{g}} P_{L} q+\bar{q} P_{R} \tilde{g} \tilde{q}_{L}-\tilde{q}_{R}^{\dagger} \overline{\tilde{g}} P_{R} q-\bar{q} P_{L} \tilde{g} \tilde{q}_{R}\right), \\
& \mathcal{L}_{m}=-m \bar{q} q-m^{2}\left(\left|\tilde{q}_{L}\right|^{2}+\left|\tilde{q}_{R}\right|^{2}\right), \\
& \mathcal{L}_{\text {soft }}=-\frac{1}{2} \tilde{m}_{\tilde{g}} \overline{\tilde{g}}\left(P_{L} \hat{f}+P_{R} \hat{f}^{\dagger}\right) \tilde{g}+\mathcal{O}\left(a, a^{\dagger}, \chi\right) \\
& -\left(\begin{array}{cc}
\tilde{q}_{L}^{\dagger} & \tilde{q}_{R}^{\dagger}
\end{array}\right)\left(\begin{array}{cc}
|\hat{f}|^{2} \tilde{M}_{L}^{2} & m \hat{f}^{\dagger} \tilde{M}_{L R} \\
m \hat{f} \tilde{M}_{L R} & |\hat{f}|^{2} \tilde{M}_{R}^{2}
\end{array}\right)\left(\begin{array}{c}
\tilde{q}_{L} \\
\tilde{q}_{R}
\end{array}\right) .
\end{aligned}
$$

In the soft-breaking terms we have not written out the explicit form of the terms containing the $a, \chi$ components of the chiral supermultiplet we use to generate the soft breaking. The usual breaking terms are obtained by setting $\hat{f}$ to the constant $f_{0}$.
The parts of the Lagrangian containing the ghosts and the external fields are $\mathcal{L}_{\text {fix }}$, gh as given in eq. (3) and

$$
\begin{aligned}
\mathcal{L}_{\mathrm{ext}}= & Y_{G}{ }_{a}^{\mu} s G_{a \mu}+\overline{\tilde{y}_{\tilde{g}}} s \tilde{g}+Y_{c} s c \\
& +\tilde{y}_{L} s \tilde{q}_{L}+\left(s \tilde{q}_{L}^{\dagger}\right) \tilde{y}_{L}^{\dagger}+\tilde{y}_{R} s \tilde{q}_{R} \\
& +\left(s \tilde{q}_{R}^{\dagger}\right) \tilde{y}_{R}^{\dagger}+\bar{y} s q-(s \bar{q}) y, \\
\mathcal{L}_{\mathrm{bil}}= & \frac{1}{2}\left(\bar{y}_{\tilde{g} a}\left(P_{R}-P_{L}\right) \epsilon\right)\left(\bar{\epsilon}\left(P_{R}-P_{L}\right) \tilde{y}_{\tilde{g} a}\right) \\
& -2\left(\bar{y} P_{R} \epsilon\right)\left(\bar{\epsilon} P_{L} y\right)-2\left(\bar{y} P_{L} \epsilon\right)\left(\bar{\epsilon} P_{R} y\right) .
\end{aligned}
$$

Then the classical action is the sum of these parts:

$$
\begin{aligned}
\Gamma_{\mathrm{cl}}= & \int d^{4} x\left(\mathcal{L}_{\mathrm{kin}}+\mathcal{L}_{m}+\mathcal{L}_{\mathrm{soft}}+\mathcal{L}_{\mathrm{fix}, \mathrm{gh}}+\mathcal{L}_{\text {ext }}+\mathcal{L}_{\mathrm{bil}}\right) \\
& +\mathcal{O}\left(a, a^{\dagger}, \chi\right) .
\end{aligned}
$$

It satisfies all defining symmetry requirements of sec. 2.4 .

\section{B Feynman rules}

In this section we give a list of the Feynman rules we need in our calculations, in particular of the ones involving the external $Y$ fields and the $\epsilon$ ghosts.

- We take all momenta as incoming.

- The $\epsilon$ ghosts are space-time independent constants and do not carry a momentum.

- Many of the following Feynman rules have to be used with different orderings of the fermions. In the case of fermionic spinors the flipping rules of 14 have to be applied, and in the case of fermionic scalars and vectors the orderings correspond to different global signs of the vertices. For ease of reference we give the alternative rules explicitely for the cases we will need later.

For brevity, we write $\Gamma$ instead of $\Gamma_{\mathrm{cl}}$ for the classical action in this section.

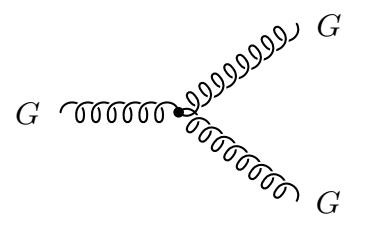

$$
\begin{gathered}
i \Gamma_{G_{a}^{\rho} G_{b}^{\mu} G_{c}^{\nu}}\left(p_{a}, p_{b}, p_{c}\right) \\
=-g f_{a b c}\left[g_{\rho \mu}\left(p_{a}-p_{b}\right)_{\nu}\right. \\
+g_{\mu \nu}\left(p_{b}-p_{c}\right)_{\rho} \\
\left.+g_{\nu \rho}\left(p_{c}-p_{a}\right)_{\mu}\right]
\end{gathered}
$$

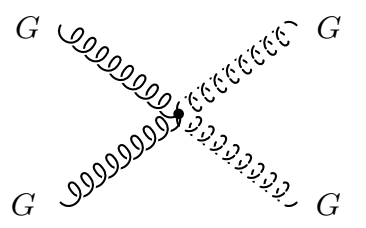

$$
\begin{aligned}
& i \Gamma_{G_{a}^{\mu} G_{b}^{\nu} G_{e}^{\rho} G_{f}^{\sigma}} \\
= & -i g^{2}\left[f_{a b c} f_{e f c}\left(g_{\mu \rho} g_{\sigma \nu}-g_{\mu \sigma} g_{\nu \rho}\right)\right. \\
& +f_{a e c} f_{f b c}\left(g_{\mu \sigma} g_{\nu \rho}-g_{\mu \nu} g_{\rho \sigma}\right) \\
& \left.+f_{a f c} f_{b e c}\left(g_{\mu \nu} g_{\rho \sigma}-g_{\mu \rho} g_{\sigma \nu}\right)\right]
\end{aligned}
$$



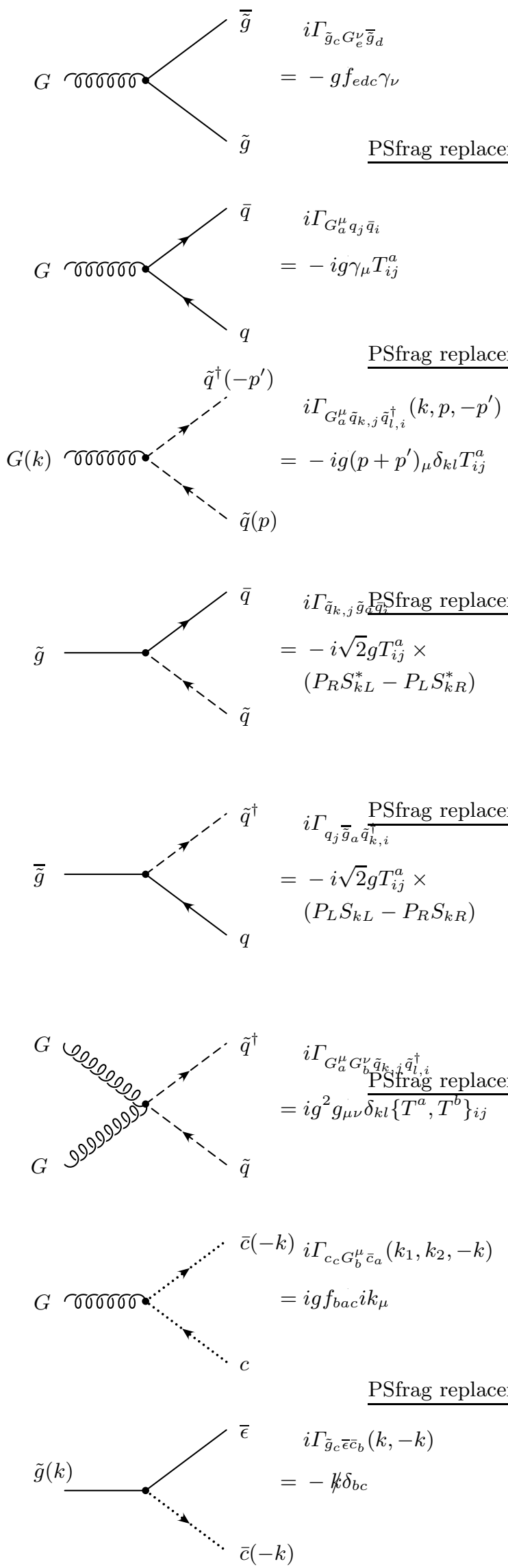
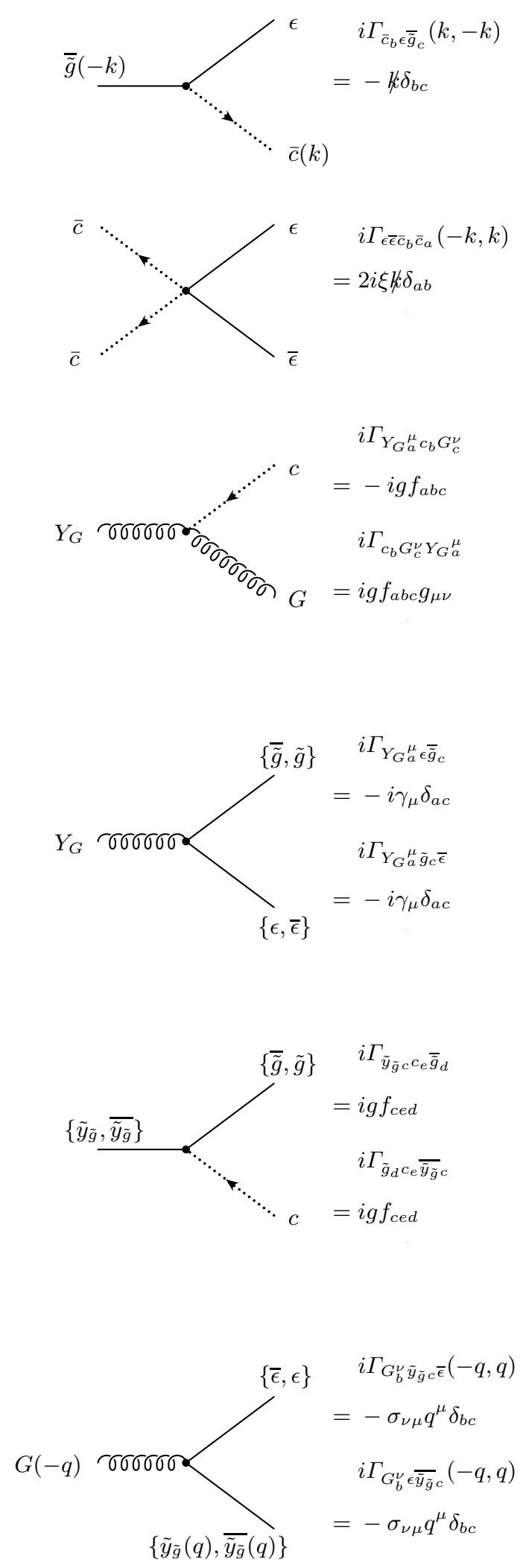


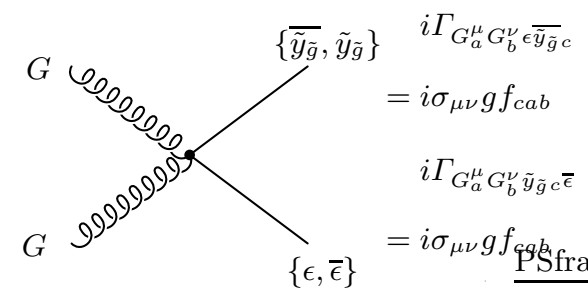

$\{\bar{\epsilon}, \epsilon\}$
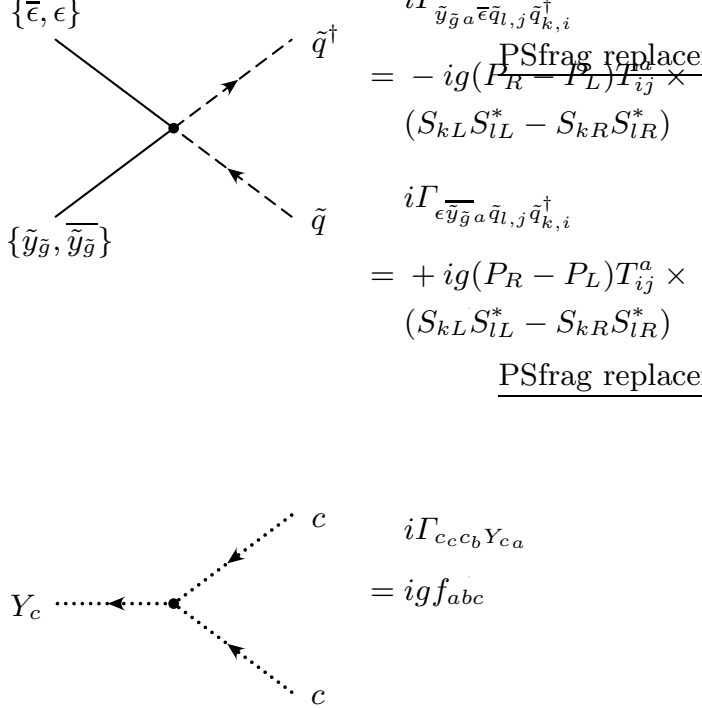

$\bar{\epsilon}$
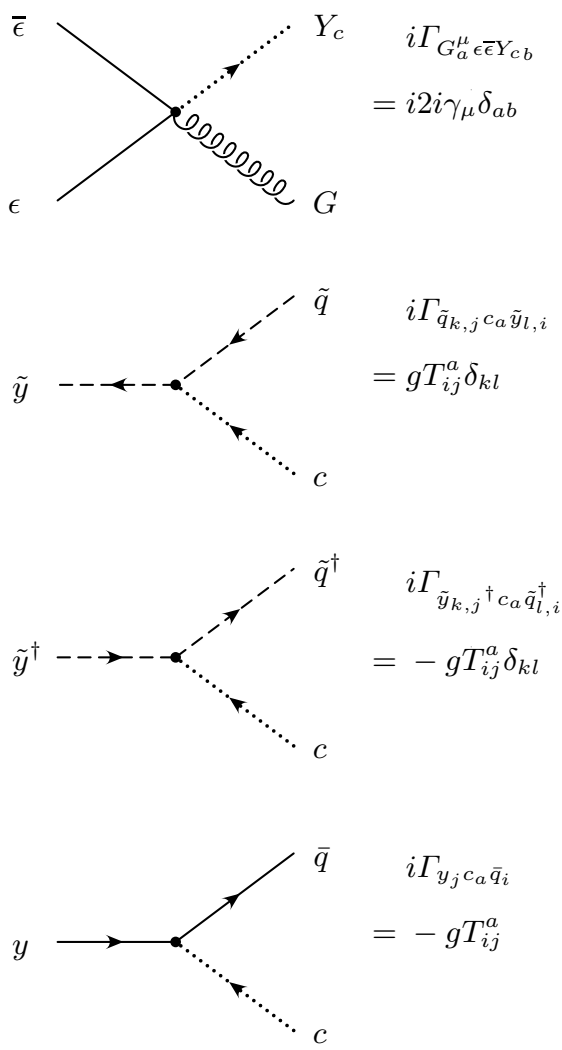
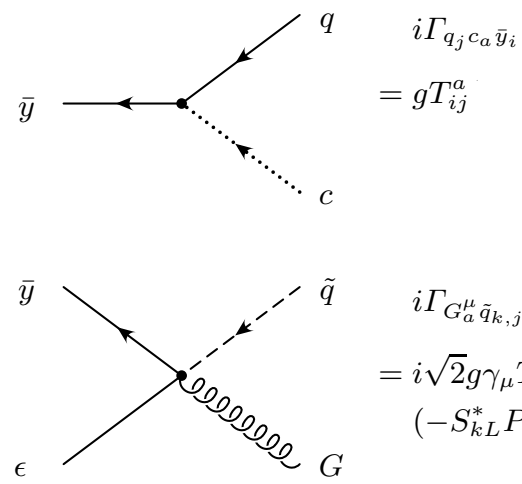

$$
\begin{aligned}
& i \Gamma_{G_{a}^{\mu} \tilde{q}_{k, j} \epsilon \bar{y}_{i}} \\
= & i \sqrt{2} g \gamma_{\mu} T_{i j}^{a} \times \\
& \left(-S_{k L}^{*} P_{L}+S_{k R}^{*} P_{R}\right)
\end{aligned}
$$

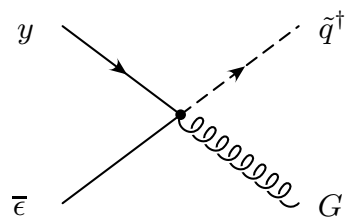

$$
\begin{aligned}
& i \Gamma_{G_{a}^{\mu} y_{j} \tilde{q}_{k, i}^{\dagger} \bar{\epsilon}^{\dagger}} \\
= & i \sqrt{2} g \gamma_{\mu} T_{i j}^{a} \times \\
& \left(S_{k L} P_{R}-S_{k R} P_{L}\right)
\end{aligned}
$$

\section{One-loop results}

In the following we give a list of the one-loop Feynman diagrams and results for the vertex functions corresponding to symmetry transformations, i.e. vertex functions involving external $Y$ sources and ghost fields. We use dimensional regularization with an anticommuting $\gamma_{5}$ or dimensional reduction and use the variable $\theta_{\text {DReg }}$ to distinguish both results. It takes the value $\theta_{\mathrm{DReg}}=1$ in the case of dimensional regularization and $\theta_{\text {DReg }}=0$ in the case of dimensional reduction. 9 We specify the results in the limit of infinite momenta, which is sufficient for our purposes. There all masses and the subleading momentum dependence can be neglected. In this subsection $\mathcal{O}\left(p^{n}\right)$ denotes a momentum dependence of the form $p^{n} \times$ powers of $\log p$. The vertex functions involving only physical fields can be calculated using standard methods, so they are not displayed here.

The one-loop functions appearing are defined in App. D and have the arguments

$$
\begin{aligned}
& B_{0}=B_{0}\left(p^{2}, 0,0\right), \\
& C_{0}=C_{0}\left(p^{2},(p+k)^{2}, k^{2}, 0,0,0\right), \\
& C_{1}=C_{1}\left(p^{2}, p^{2}, 0,0,0,0\right),
\end{aligned}
$$

\footnotetext{
9 Here a word to the consistency of the schemes is in order. Both dimensional reduction and dimensional regularization in the way we use it are mathematically inconsistent and cannot be used at all orders. An inconsistent scheme can yield incorrect results if imaginary or non-local contributions turn out to be wrong, because this violates unitarity or causality. Here, however, it is easy to see that the difference of our schemes to a consistent one like the prescription of 13 is a sum of local counterterms, and the results obtained using the scheme of 13. with appropriate counterterms would coincide with ours. Therefore, our results are correct.
}

10 The Feynman gauge $\xi=1$ is used. 
where $p$ is the momentum argument of the corresponding vertex function. Furthermore, as explained in sec. 3.1 we introduce non-symmetric counterterms $\delta_{i}$ to all vertex functions except for the self energies and the $\tilde{q} \tilde{q} G_{\mu}$ interaction. These counterterms have to be chosen in such a way that the Slavnov-Taylor identity is satisfied.

\section{C.1 Vertex functions involving $Y_{G}{ }^{\mu}, \tilde{y}_{\tilde{g}}, Y_{c}$}

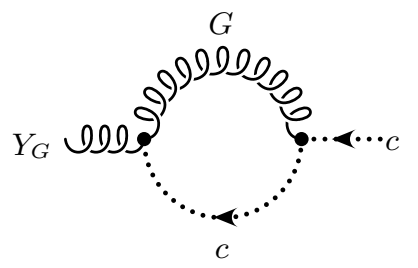

Fig. 1. The one-loop diagram contributing to the vertex function $\Gamma_{Y_{G}} \mu_{c}$.

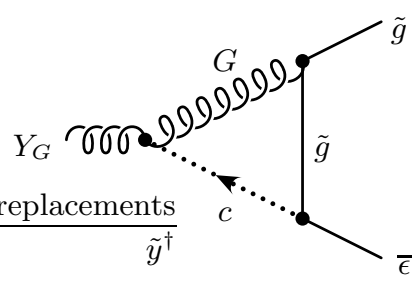

Fig. 2. The one-loop diagram contributing to the vertex function $\Gamma_{Y_{G}}{ }^{\tilde{g}} \bar{\epsilon}$.
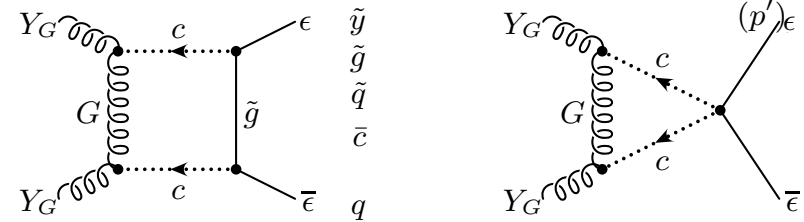

Fig. 4. The one-loop diagrams contributing to the vertex function $\Gamma_{Y_{G}{ }^{\mu} Y_{G}{ }^{\nu} \epsilon \bar{\epsilon}}$.

$$
\begin{aligned}
& \Gamma_{Y_{G a}^{\mu} c_{c}}(q,-q)=-i q_{\mu} \delta_{a c} \times \\
& \quad\left(1+\delta_{c Y_{G}}-\frac{\alpha_{s} C(A)}{4 \pi} \frac{1}{2} B_{0}\right), \\
& \Gamma_{Y_{G} a \epsilon \bar{g}_{c}}=-\gamma_{\mu} \delta_{a c}\left(1+\delta_{Y_{G} \tilde{g} \epsilon}+\frac{\alpha_{s} C(A)}{4 \pi} B_{0}\right), \\
& \Gamma_{Y_{G a}^{\mu} \tilde{g}_{c} \bar{\epsilon}}=-\gamma_{\mu} \delta_{a c}\left(1+\delta_{Y_{G} \tilde{g} \epsilon}+\frac{\alpha_{s} C(A)}{4 \pi} B_{0}\right), \\
& \Gamma_{\epsilon \bar{\epsilon} Y_{G}{ }_{a}^{\mu} Y_{G_{c}^{\nu}}^{\nu}}=\mathcal{O}\left(p^{-2}\right),
\end{aligned}
$$

$$
\begin{aligned}
& \Gamma_{G_{b}^{\nu} \tilde{y}_{\tilde{g}} \bar{\epsilon}}(-q, q)=i \sigma_{\nu \mu} q^{\mu} \delta_{b c} \times \\
& \left(1+\delta_{G \epsilon \tilde{y} \tilde{g}}-\frac{\alpha_{s} C(A)}{4 \pi} B_{0}\right), \\
& \Gamma_{G_{b}^{\nu} \epsilon \overline{\tilde{y} \tilde{g}} c}(-q, q)=i \sigma_{\nu \mu} q^{\mu} \delta_{b c} \times \\
& \left(1+\delta_{G \epsilon \tilde{y} \tilde{g}}-\frac{\alpha_{s} C(A)}{4 \pi} B_{0}\right), \\
& \Gamma_{G_{a}^{\mu} G_{b}^{\nu} \tilde{y} \tilde{g} d \bar{\epsilon}}(-q, 0, q)=-\frac{1}{2} i g f_{a b d} \times \\
& \left(\left(4 \frac{C(A) \alpha_{s}}{4 \pi} q^{2} B_{0}^{\prime}+2+2 \delta_{\tilde{y}_{\tilde{g}} \epsilon G G}\right) g_{\mu \nu}\right. \\
& -\left(3 \frac{C(A) \alpha_{s}}{4 \pi} q^{2} B_{0}^{\prime}+2+2 \delta_{\tilde{y} \tilde{g} \epsilon G G}\right) \gamma_{\mu} \gamma_{\nu} \\
& \left.-\frac{C(A) \alpha_{s}}{4 \pi} B_{0}^{\prime}\left(2 \gamma_{\nu} \not q q_{\mu}+2 q_{\nu} q_{\mu}-3 \gamma_{\mu} \not q q_{\nu}\right)\right) \text {, } \\
& \Gamma_{G_{b}^{\nu} \tilde{g}_{c} Y_{G}^{\rho}{ }_{d}^{\bar{\epsilon}}}(0,-q, q)=\frac{i}{2} \frac{C(A) \alpha_{s}}{4 \pi} g B_{0}^{\prime} f_{c b d} \times \\
& \left(\not q g_{\nu \rho}-\gamma_{\nu} \phi \gamma_{\rho}-3 \gamma_{\rho} q_{\nu}+\gamma_{\nu} q_{\rho}\right) \\
& \Gamma_{G_{b}^{\nu} \epsilon \bar{\epsilon} Y_{c}}=2 i \gamma_{\nu}\left(1+\delta_{G \epsilon \bar{\epsilon} Y_{c}}+\frac{\alpha_{s} C(A)}{4 \pi} \frac{1}{2}\left(B_{0}\right)\right) \text {. }
\end{aligned}
$$

\section{C.2 Vertex functions involving $\tilde{y}, y$}
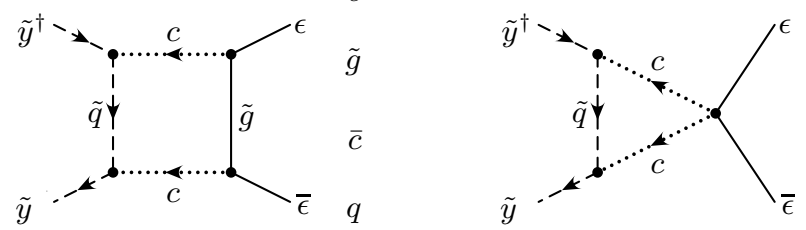

Fig. 10. The one-loop diagrams contributing to the vertex function $\Gamma_{\tilde{y} \tilde{y}} \bar{\epsilon}_{\epsilon}$.

$$
\begin{aligned}
& \Gamma_{\tilde{y}_{k, j}^{\dagger} \epsilon \bar{q}_{i}}=\sqrt{2}\left(S_{k L}^{*} P_{R}-S_{k R}^{*} P_{L}\right) \delta_{i j} \times \\
& \quad\left(1+\delta_{\tilde{y} \epsilon q}+\frac{\alpha_{s} C(F)}{4 \pi} B_{0}\right) \\
& \Gamma_{q_{i} \bar{\epsilon} \tilde{y}_{k, j}}=\sqrt{2}\left(S_{k L} P_{L}-S_{k R} P_{R}\right) \delta_{i j} \times \\
& \quad\left(1+\delta_{\tilde{y} \epsilon q}+\frac{\alpha_{s} C(F)}{4 \pi} B_{0}\right) \\
& \Gamma_{\tilde{q}_{k, j} \epsilon \bar{y}_{i}}(q,-q)=\sqrt{2} \not q\left(S_{k L}^{*} P_{R}-S_{k R}^{*} P_{L}\right) \delta_{i j} \times \\
& \quad\left(1+\delta_{y \tilde{q} \epsilon}-\frac{\alpha_{s} C(F)}{4 \pi}\left(B_{0}\right)\right) \\
& \Gamma_{\tilde{q}_{k, j}^{\dagger} y_{i} \bar{\epsilon}}(-q, q)=\sqrt{2} \not\left(S_{k L} P_{R}-S_{k R} P_{L}\right) \delta_{i j} \times \\
& \quad\left(1+\delta_{y \tilde{q} \epsilon}-\frac{\alpha_{s} C(F)}{4 \pi}\left(B_{0}\right)\right) \\
& \Gamma_{\tilde{q}_{L} \tilde{g}_{a} \bar{\epsilon} \tilde{y}_{L}}(p, k,-p-k)=g \not p T^{a} \frac{\alpha_{s}}{4 \pi} \times \\
& \quad\left(\frac{C(A)}{2}\left(2 C_{0}+\left(1-2 P_{L}\right) C_{1}\right)\right.
\end{aligned}
$$



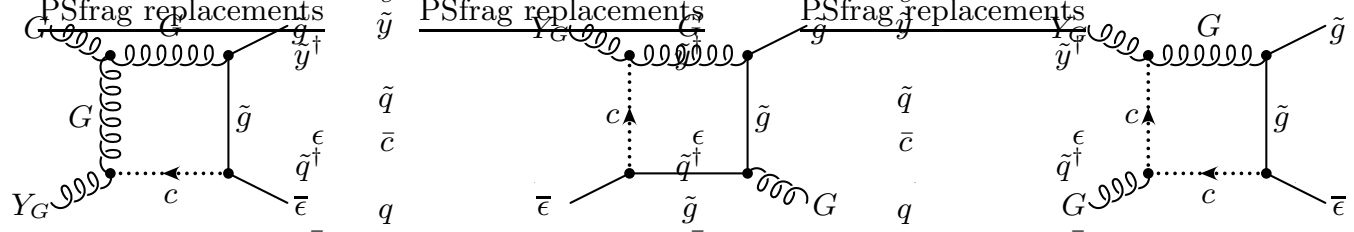

Fig. 3. The one-loop diagrams contributing to the vertex function $\Gamma_{G^{\mu}} \tilde{g} \bar{\epsilon} Y_{G}^{\nu}$.
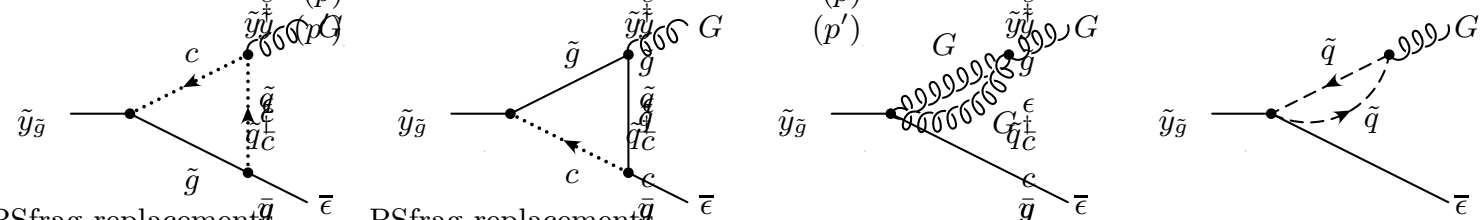

Fig. 5. The one-loop diagrams contributing to the vertex function $\Gamma_{G^{\nu}} \tilde{y} \tilde{g} \bar{\epsilon}$.
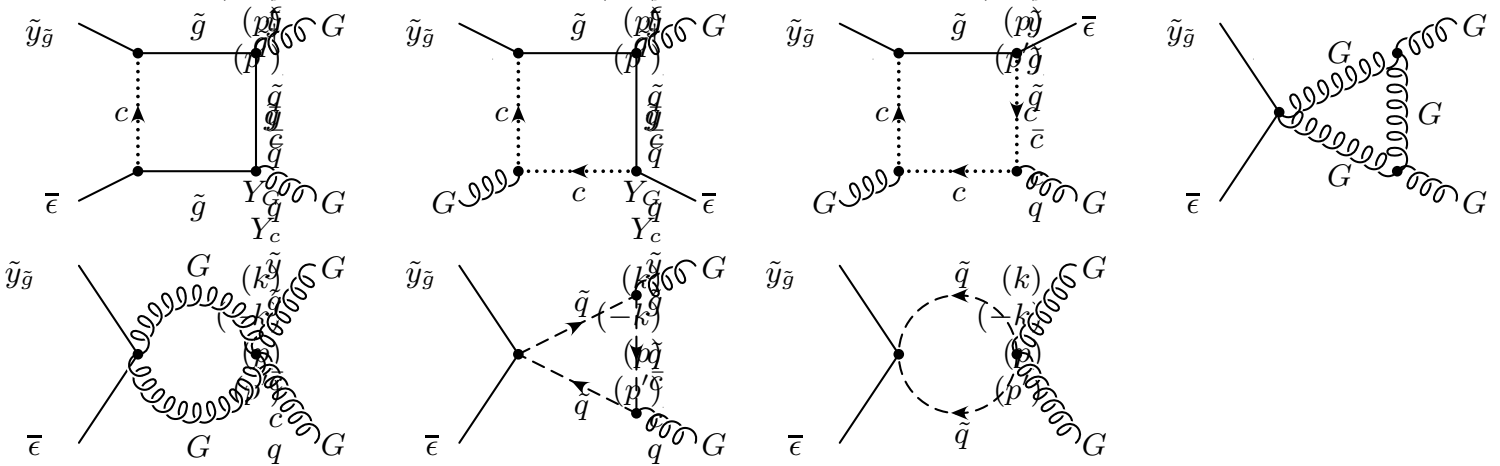

Fig. 6. The one-loop diagrams contributing to the vertex function $\Gamma_{G^{\mu} G^{\nu}} \tilde{y}_{\tilde{g}} \bar{\epsilon}$.

$$
\begin{aligned}
& \left.+C(F)\left(2 P_{L} C_{1}\right)\right)+\mathcal{O}\left(k^{-1}\right) \\
& \Gamma_{\tilde{q}_{L}^{\dagger} \tilde{g}_{a} \bar{\epsilon} \tilde{y}_{L}^{\dagger}}(-p, k, p-k)=-g \not p T^{a} \frac{\alpha_{s}}{4 \pi} \times \\
& \left(\frac{C(A)}{2}\left(2 C_{0}+\left(1-2 P_{R}\right) C_{1}\right)\right. \\
& \left.+C(F)\left(2 P_{R} C_{1}\right)\right)+\mathcal{O}\left(k^{-1}\right) \\
& \Gamma_{\tilde{y}_{j} \epsilon \bar{\epsilon} \tilde{y}_{i}^{\dagger}}(q,-q)=\mathcal{O}\left(q^{-2}\right) .
\end{aligned}
$$

\section{C.3 Identities involving $\omega^{\mu}$}

Owing to the non-renormalization of the terms involving $\omega^{\mu}$ (see sec. 2.4) we have

$$
\begin{aligned}
\Gamma_{\tilde{q}_{j} \tilde{y}_{i} \omega^{\mu}}(q,-q) & =\delta_{i j} q_{\mu}, \\
\Gamma_{G_{a}^{\rho} Y_{G_{b}} \omega^{\mu}}(q,-q) & =g_{\rho \sigma} \delta_{a b} q_{\mu}, \\
\frac{\delta^{2} s \omega^{\mu}}{\delta \epsilon \delta \bar{\epsilon}} & =2 \gamma^{\mu} .
\end{aligned}
$$

\section{One-loop functions}

We use the following one-loop two- and three-point functions [15]:

$$
\begin{aligned}
B_{0} & =\int \frac{1}{\left[k^{2}-m_{0}^{2}\right]\left[\left(k+p_{1}\right)^{2}-m_{1}^{2}\right]}, \\
C_{\{0, \mu\}} & =\int \frac{\left\{1, k_{\mu}\right\}}{\left[k^{2}-m_{0}^{2}\right]\left[\left(k+p_{1}\right)^{2}-m_{1}^{2}\right]\left[\left(k+p_{2}\right)^{2}-m_{2}^{2}\right]},
\end{aligned}
$$

with

$$
\int \rightarrow \mu^{4-D} \frac{16 \pi^{2}}{i} \int \frac{d^{D} k}{(2 \pi)^{D}}
$$

and the tensor decomposition

$$
\begin{aligned}
C_{\mu} & =p_{1 \mu} C_{1}+p_{2 \mu} C_{2} \\
B_{0} & =B_{0}\left(p_{1}^{2}, m_{0}^{2}, m_{1}^{2}\right) \\
C_{i j} & =C_{i j}\left(p_{1}^{2},\left(p_{2}-p_{1}\right)^{2}, p_{2}^{2}, m_{0}^{2}, m_{1}^{2}, m_{2}^{2}\right)
\end{aligned}
$$

in the conventions of 16,17 .

\section{E Useful formulas}



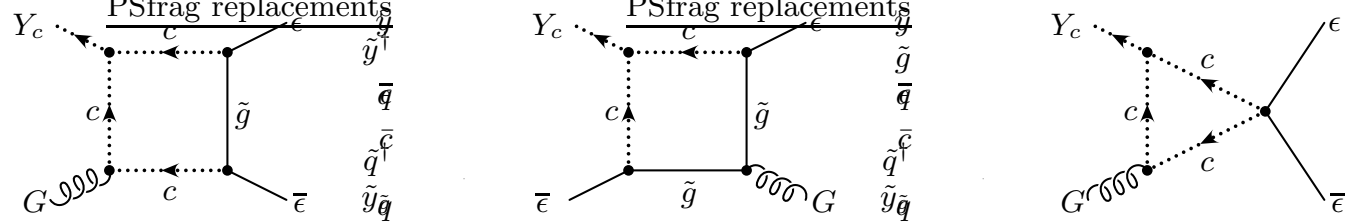

Fig. 7. The one-loop diagrams contributing to the vertex function $\Gamma_{G^{\nu}} \epsilon \bar{\epsilon} Y_{c}$.
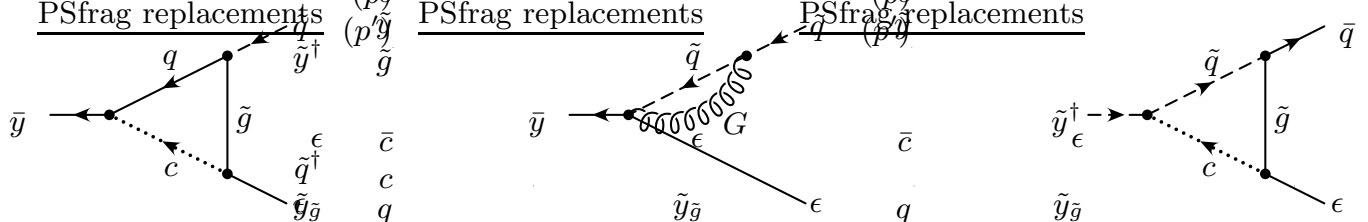

Fig. 8. The one-loop diagrams contributing to the vertex functions $\Gamma_{\tilde{q} \epsilon \bar{y}}$ and $\Gamma_{\epsilon \bar{q} \tilde{y}^{\dagger}}$.
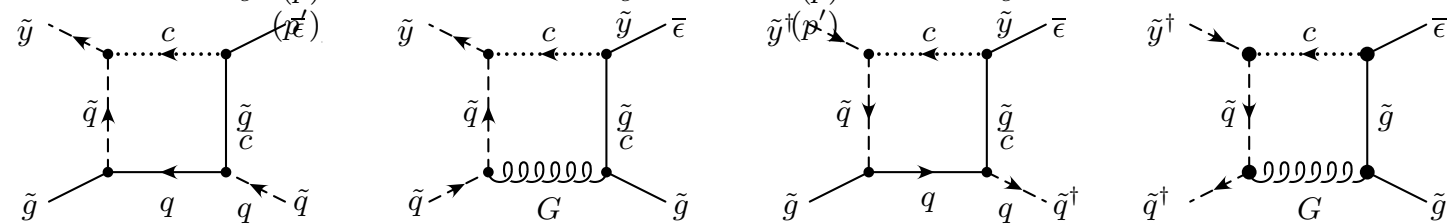

Fig. 9. The one-loop diagrams contributing to the vertex functions $\Gamma_{\tilde{q} \tilde{g} \bar{\epsilon} \tilde{y}}$ and $\Gamma_{\tilde{q}^{\dagger} \tilde{g} \bar{\epsilon} \tilde{y}^{\dagger}}$.

E.1 $S U(3)$

$$
\begin{aligned}
& {\left[T^{a}, T^{b}\right]=i f_{a b c} T^{c},} \\
& f_{a b c} f_{d b c}=C(A) \delta_{a d}, \quad C(A)=3, \\
& \operatorname{Tr}\left(T^{a} T^{b}\right)=T(F) \delta_{a b}, \quad T(F)=\frac{1}{2}, \\
& \left(T^{a} T^{a}\right)_{i j}=C(F) \delta_{i j}, \quad C(F)=\frac{4}{3} .
\end{aligned}
$$

\section{E.2 Spinor identities}

In $\mathcal{L}_{\text {ext }}$ and the Slavnov-Taylor operator several useful replacements are possible. The signs are due to the bosonic statistics of the external spinors $\tilde{y}_{\tilde{g}}, y$ :

$$
\begin{gathered}
\overline{\tilde{y}_{\tilde{g}}} s \tilde{g}=-(s \overline{\tilde{g}}) \tilde{y}_{\tilde{g}} \\
\frac{\delta \Gamma}{\delta \tilde{g}_{a}} \frac{\delta \Gamma}{\delta \tilde{y}_{\tilde{g} a}}=-\frac{\delta \Gamma}{\delta \tilde{y}_{\tilde{g} a}} \frac{\delta \Gamma}{\delta \overline{\tilde{g}}_{a}} \\
\frac{\delta \Gamma}{\delta q} \frac{\delta \Gamma}{\delta \bar{y}}=-\frac{\delta \Gamma}{\delta y^{C}} \frac{\delta \Gamma}{\delta \bar{q}^{C}} \\
\frac{\delta \Gamma}{\delta y} \frac{\delta \Gamma}{\delta \bar{q}}=-\frac{\delta \Gamma}{\delta q^{C}} \frac{\delta \Gamma}{\delta \bar{y}^{C}} .
\end{gathered}
$$

\section{References}

1. W. Siegel, Phys. Lett. B84 (1979) 193.

2. W. Siegel, Phys. Lett. B94 (1980) 37.

3. D.M. Capper, D.R.T. Jones, P. van Nieuvenhuizen, Nucl. Phys. B167 (1980) 479.
4. I. Jack, D.R.T. Jones, K.L. Roberts, Z. Phys. C62 (1994) 161, Z. Phys. C63 (1994) 151.

5. P. Breitenlohner, D. Maison, "Renormalization of supersymmetric Yang-Mills theories", in Cambridge 1985, Proceedings: "Supersymmetry and its applications", p. 309.

6. P.L. White, Class. Quantum Grav. 9 (1992) 1663.

7. N. Maggiore, O. Piguet, S. Wolf, Nucl. Phys. B458 (1996) 403.

8. W. Hollik, E. Kraus, D. Stöckinger, Eur. Phys. J. C11(1999) 365.

9. N. Maggiore, O. Piguet, S. Wolf, Nucl. Phys. B476 (1996) 329.

10. W. Hollik, E. Kraus, D. Stöckinger, hep-ph/0007134.

11. S.P. Martin, M.T. Vaughn, Phys. Lett. B318 (1993) 331; W. Beenakker, R. Höpker, P.M. Zerwas, Phys. Lett. B378 (1996) 159.

12. W. Beenakker, R. Höpker, M. Spira, P.M. Zerwas, Nucl. Phys. B492 (1997) 51.

13. P. Breitenlohner, D. Maison, Comm. Math. Phys. 52 (1977) 11, 39, 55.

14. A. Denner, H. Eck, O. Hahn, J. Küblbeck, Nucl. Phys. B387 (1992) 467.

15. G. Passarino, M. Veltman, Nucl. Phys. B160 (1979) 151.

16. T. Hahn, Loop Tools User's Guide, ww. feynarts.de/looptools.

17. A. Denner, Fortschr. Phys. 41 (1993) 307. 